\title{
Inefficiencies in Networked Markets ${ }^{\dagger}$
}

\author{
By Matthew Ellioto*
}

\begin{abstract}
In many markets, relationship specific investments are necessary for trade. These formed relationships constitute a networked market in which not all buyers can trade with all sellers. We show that networked markets can be decomposed to identify how alternative trading opportunities affect who trades with whom and at what price. This uncovers agents' incentives to invest in relationships. Investment inefficiencies can eliminate all the gains from trade, but for reasons that differ depending on how investments are made. Three applications are considered in detail: high-skill labor markets, merger markets when industries are consolidating, and the international market for natural gas. (JEL C78, D85, D86)
\end{abstract}

$\mathrm{n}$ many markets a buyer and a seller must make a relationship-specific investment before they can trade: Countries invest in pipelines to trade oil and natural gas; contractors learn the specific requirements of manufacturers; ${ }_{1}^{1}$ acquirers pay due diligence costs, while takeover targets reveal private information; there are interviews prior to recruitment; art dealers authenticate paintings; illegal-goods handlers risk transacting with under-cover law enforcers; etc. After these investments have been made, only some buyer-seller pairs can trade with each other-the market is networked. This paper analyzes inefficiencies in these investments when long-term contracts cannot be enforced.

To analyze inefficiencies in the investments that enable trade it is necessary to first understand how the structure of a formed networked market affects bargained outcomes, who trades with whom, and at what prices. Letting the buyers and sellers be the nodes, links in a network can be used to represent which buyer-seller pairs have made the required investments to trade with each other. We consider bargaining outcomes in which no two linked agents have a profitable pairwise deviation. By utilizing and extending results from the matching literature, an intuitive algorithm is constructed that decomposes any network to identify: (i) the role of each link in the

\footnotetext{
* Faculty of Economics, University of Cambridge and Division of the Humanities and Social Sciences, California Institute of Technology, Pasadena, CA 91125 (e-mail: melliott@ hss.caltech.edu). I am grateful to Matt Jackson and Muriel Niederle for extensive discussions, advice and guidance and to Rachel Kranton for very helpful comments on an earlier draft. I also thank anonymous referees, Manuel Amador, Attila Ambrus, Doug Bernheim, Francis Bloch, Itay Fainmesser, Guillaume Fréchette, Sanjeev Goyal, Bob Hall, Fuhito Kojima, Pete Kyle, Jon Levin, Paul Milgrom, David Myatt, Michael Ostrovsky, Al Roth, Ilya Segal, Jan-Peter Siedlarek, Andy Skrzypacz, and Fernando Vega-Redondo, as well as numerous seminar and conference participants, for valuable feedback. I gratefully acknowledge financial support from the Sonja and William Davidow Fellowship administered through the Stanford Institute for Economic Policy Research.

Go to http://dx.doi.org/10.1257/mic.20130098 to visit the article page for additional materials and author disclosure statement(s) or to comment in the online discussion forum.

${ }^{1}$ An example is the Japanese engineering and electronics industry (Nishiguchi 1994).
} 
network - whether it is used for trade or to generate an outside option and affect the terms of trade; and (ii) the effect of each link on each agent's bargained outcome. This network decomposition clarifies agents' investment incentives and underlies the subsequent analysis of investment inefficiencies.

Relationship-specific investments are subject to well-understood inefficiencies. An agent may fail to make an efficient up-front investment, anticipating that he will be held up and unable to recover his costs. This problem can lead to underinvestment. An agent may also make costly investments into alternative trading relationships just to improve his terms of trade. This overinvestment generates no additional gains from trade and is also inefficient. We provide a systematic treatment of these inefficiencies when investments are made to enable trade in a market context. Which agents will make the required investments to trade? Who will actually trade with whom? How large will inefficiencies be? Which types of inefficiency will be most severe and when?

We consider two investment protocols. In protocol A, both the buyer and seller must make fixed, separate investments before they can trade, and we show that for this protocol the inefficiency problem of most concern is the hold-up problem. For any parametrization of the model, we characterize a tight bound on overinvestment inefficiency as buyers' and sellers' gains from trade are varied. For different parameter values, this bound ranges between 0 and 50 percent of the potential gains from trade. Underinvestment inefficiency, in contrast, may consume all the gains from trade. In protocol $\mathrm{B}$, the investment costs necessary for forming a relationship are transferable and can be split in any way between the buyer and seller. For this protocol we show that shares of the investment costs can always be found to overcome the hold-up problem - the investment share of the agent at risk of being held up can be reduced until participation is profitable. Are the relationships that are formed then efficient, or close to efficient? While it is easier to form links that will be used for trade when investments are negotiated, it is also easier to form links to establish outside options that will not be used for trade. Although protocol B overcomes the hold-up problem, it exacerbates the overinvestment problem. With negotiated joint investments, overinvestment can consume all the gains from trade.

Both investment protocols seem important in practice. In some markets the relationship-specific investments that enable trade are naturally separate and nonsubstitutable, while in others the share of investments each agent is responsible for is negotiated. We examine three applications in detail. In high-skill labor markets, interviews are required before a firm can employ a worker. Investments into interviews are nonsubstitutable, and we argue that protocol A applies. Moreover, we make the case that firms have higher bargaining power and pay the majority of the interview costs. For this parameterization of the model, the scope for underinvestment is limited (although the losses can be large when it occurs) and the magnitude of overinvestment inefficiency is bounded fairly tightly. A second application we consider is industry consolidation, where due diligence must be completed before credible offers can be made. The widespread use of termination fees ${ }^{2}$ provides a

\footnotetext{
${ }^{2}$ These are lump-sum transfers made from the target to a potential acquirer following a failed bid.
} 
mechanism through which transfers are made, and means that protocol B fits this application better. In the context of the model, we examine the Omnicare, Inc. v. NCS Healthcare, Inc. decision that ruled against the use of an exclusive-dealing agreement. We argue that by expanding the contracting space such agreements can help overinvestment be avoided. The final application we consider is the international market for natural gas, where pipelines must be constructed before trade is possible. We again argue that protcol B fits and identify possible instances of overinvestment.

This paper contributes primarily to the growing literatures on trade within, and the formation of, networked markets. The cooperative game theoretic approach we take to modeling trade builds on the matching literature and is most closely related to Kranton and Minehart (2000a). ${ }^{3}$ The main contribution to this literature is the network decomposition algorithm, which identifies how different trading opportunities affect market outcomes. Our cooperative approach yields the same solution Corominas-Bosch (2004) finds in her noncooperative bargaining model. ${ }^{4}$ However, the Corominas-Bosch approach is only applied to environments with homogeneous gains from trade-and cannot be readily extended to environments with heterogeneous gains from trade. Experimental evidence supports her theory and, by extension, our theory (Charness, Corominas-Bosch, and Fréchette 2007).

A key feature of the bargaining approach we take is that players have endogenous disagreement payoffs or "outside options," and these depend on the network structure. A related and complementary literature modeling input markets endogenizes disagreement payoffs in a different way. Building on the foundations laid in Horn and Wolinsky (1988), Iozzi and Valletti (2014) consider a supplier who simultaneously bargains bilaterally with multiple downstream producers over input prices. Suppose competition downstream is in quantities. If an agreement is not reached with a producer, the supplier may be able to switch some additional supply to their other producers at the agreed-upon price, as these producers will now demand more. When this is possible, the supplier's outside option is better and they negotiate a higher input price. In contrast, we turn off potential product market effects by assuming that the surplus a buyer and seller generate does not depend on the trades of other buyer-seller pairs. This allows us to consider general networked markets, with multiple suppliers and multiple producers, permitting an analysis of the network formation problem. How substantial product market effects can be depends on how competitive the product market is and how much producers rely on a single supplier. ${ }^{5}$

There is a large literature on inefficient investment due to hold-up problems and overinvestment in outside options. ${ }^{6}$ Unlike much of this literature, we analyze investments in a market context with multiple buyers and multiple sellers, and where

\footnotetext{
${ }^{3}$ Nava (2015) models quantity competition in networked markets and Blume et al. (2009) model trade through intermediaries that quote bid and ask prices, while Fainmesser (2010) and Fainmesser and Goldberg (2011) study repeated trade over networks.

${ }^{4}$ Polanski (2007); Gale and Kariv (2007); Abreu and Manea (2012b, 2012a); Manea (2011); and Elliott and Nava (2014) also model noncooperative bargaining games over networks.

${ }^{5}$ Lee and Fong (2013) have an interesting recent paper that builds a dynamic model with general externalities between buyers and sellers. While the cost of this generality is the loss of analytical tractability, the paper helps draw out differences between a static and dynamic approach. In Section VID, we argue that some markets are likely to be well approximated by static models, while others will be better suited to a dynamic treatment.

${ }^{6}$ See Segal (1999) for a general treatment of inefficiencies that arise when one principal contracts with many agents in the presence of externalities.
} 
investments enable trade. In Cole, Mailath, and Postlewaite (2001a, b), investments are made in attributes valued by multiple potential trade partners, albeit at possibly different rates, and conditions are found under which investments are efficient. Felli and Roberts (2002) consider investments in quality where buyers and sellers are vertically differentiated. In both cases it is critical for the results that investments be valuable to multiple potential trade partners. Investments that enable trade are modeled by Kranton and Minehart (2001), (2000b). We generalize the Kranton and Minehart environment by permitting two-sided investments in links, heterogeneous potential gains from trade and a more general bargaining solution.

Kranton and Minehart (2001, 2000b), like Cole, Mailath, and Postlewaite (2001a, b) and Felli and Roberts (2002), focus on finding conditions under which efficient investments are made. We take a different approach by identifying different types of inefficiency and measuring the efficiency losses due to them. This is complicated by the presence of multiple stable networks. Even for given gains from trade in a very small market with 5 buyers and 5 sellers, just finding the set of stable networks is hard-there are over 30 million possible (bipartite) networks to consider. ${ }^{7}$ We make some progress with these questions by importing two concepts from the computer science literature: the cost of anarchy and the price of anarchy, which measure the efficiency loss in the best and worst stable networks, respectively (Koutsoupias and Papadimitriou 2009; Roughgarden and Tardos 2004). When bounding inefficiency losses from above, we focus on the worst stable network; and when bounding inefficiency losses from below, we focus on the best stable network. Despite taking this conservative approach, we find tight upper and lower bounds on different types of inefficiency that vary with the investment protocol. These results contrast with the positive efficiency results from the existing literature. We also depart from the existing literature by allowing investments to be negotiated. ${ }^{8}$

The paper proceeds as follows. Section I provides a simple example illustrating the main points of the paper. Section II then sets up the model, and Section III analyzes bargaining over a formed network. Section IV identifies the efficient network(s), providing a useful benchmark for the set of stable networks that are considered in Section V. Section V examines the inefficiency present in stable networks. Section VI discusses three applications of the model, and Section VII concludes.

\section{Example}

This section presents a very simple example to illustrate the inefficiency results. Suppose that there are two sellers, $s_{1}$ and $s_{2}$, and a single buyer $b_{1}$. Each seller has a single unit to sell and the buyer demands one unit. To enable trade between either seller and $b_{1}$, a specific investment must be made at cost $c=\frac{1}{2}-\varepsilon$, where $\varepsilon$ is small and positive. Neither seller directly values the good they sell, and $b_{1}$ values

\footnotetext{
${ }^{7}$ The relevant set of networks are bipartite, as buyers can trade only with sellers. For $m$ buyers and $n$ sellers, there are $2^{m n}$ such networks.

${ }^{8}$ Currarini and Morelli (2000) consider a noncooperative network formation game in which players form links and propose surplus splits, thereby endogenizing these splits. They find that the efficient network is stable under their condition of "size monotonicity."
} 
Panel A. Potential links

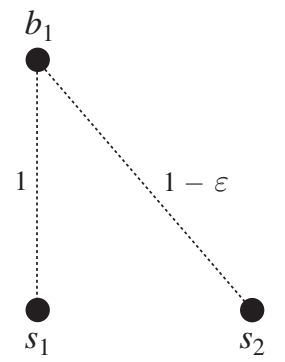

Panel B. Efficient network

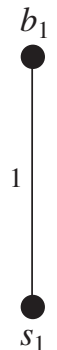

Figure 1. NETWORKED MARKET
Panel C. Complete network

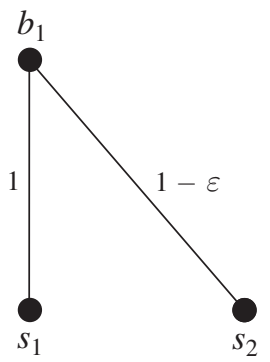

the good from $s_{1}$ at 1 and the good from $s_{2}$ at $1-\varepsilon$. Figure 1, panel A shows the potential gains from trade.

The efficient network maximizes the net gains from trade (the realized gains from trade less resources spent on forming links) and is shown in Figure 1, panel B. The net gains from trade generated by this network are $1-\left(\frac{1}{2}-\varepsilon\right)=\frac{1}{2}+\varepsilon$.

We first consider investment protocol $\mathrm{A}$ and suppose a buyer must pay $\gamma c$ toward forming a link, while a seller must pay $(1-\gamma) c$, where $\gamma \in(0,1)$ is an exogenous parameter. Let the sellers have all the bargaining power and make take-it-or-leave-it offers after a network is formed. If the efficient network were formed $b_{1}$ would have no alternative but to trade with $s_{1}$, and $s_{1}$ would sell to $b_{1}$ at a price of 1 , extracting all the surplus and leaving $b_{1}$ with a net payoff of $-\gamma c$. Anticipating this, $b_{1}$ will not invest in a single link to $s_{1}$ for fear of being held up. The complete network is not stable either. Sellers $s_{1}$ and $s_{2}$ will compete with each other to supply $b_{1}$, driving the price down until at least one of them is unable to recover their (sunk) investment. The empty network is the unique stable network. Underinvestment inefficiency due to a hold-up problem consumes all the possible gains from trade.

Consider instead protocol B, and let the buyer and sellers negotiate their investment shares. These negotiations are bilateral, and contingent contracts cannot be written. The empty network is no longer stable: $b_{1}$ and $s_{1}$ could agree to split the cost $c$ so that $s_{1}$ paid the entire cost, leaving $b_{1}$ 's payoff unaffected, but strictly increasing $s_{1}$ 's payoff. However, the efficient network is not stable either. If the efficient network were formed $s_{1}$ would extract all the rents and $b_{1}$ would receive a payoff of at most zero. Buyer $b_{1}$ would then be willing to pay the entire investment cost required to form a link to $s_{2}$. With this additional link, $s_{1}$ and $s_{2}$ would compete to supply $b_{1}$, and $b_{1}$ would extract rents $1-\varepsilon$ less his investment costs. With negotiation, the unique stable network is the complete network, with $b_{1}$ paying all the investment costs of forming the link to $s_{2}$ and at least $c-\varepsilon$ of the investment costs of forming the link with $s_{1}$.

Under protocol A, the unique stable network is the empty network, and all gains from trade are lost to underinvestment. Under protocol B, there is no longer any underinvestment. However, underinvestment inefficiency is replaced by overinvestment inefficiency. Buyer $b_{1}$ forms a link to $s_{2}$ for the sole purpose of improving his terms of trade with $s_{1}$. From an efficiency perspective, this investment generates no 
gains from trade and is a waste of resources. Furthermore, as $\varepsilon \rightarrow 0$ overinvestment inefficiency consumes all the possible gains from trade.

\section{Model}

There is a set of $m$ buyers denoted $P$ and a set of $n$ sellers denoted $Q$. The value of trade between a buyer $i$ and a seller $j$ is given by $a_{i j} \geq 0$. Each buyer demands one unit of the good, and each seller supplies one unit of the good. The $m \times n$ dimensional matrix a describes the value of all potential bilateral trades.

There are two stages. In stage one, buyers and sellers make relationship-specific investments to enable trade, where the gains from trade a are common knowledge. The resulting set of potential trading relationships is $L=\left\{l_{i j}\right\}$. As $n$ and $m$ are fixed, the network $(n, m, L)$ depends only on $L$ and abusing notation we will refer to $L$ as a network. A link is formed enabling trade between $i$ and $j\left(l_{i j} \in L\right)$ if and only if their joint investment is at least $c$; otherwise no link is formed $\left(l_{i j} \notin L\right)$. Two investment protocols are considered. In protocol A, buyers and sellers choose whether or not to pay an exogenously fixed proportion of the cost $c$ : $\gamma$ and $1-\gamma$, respectively. In protocol B, buyers and sellers negotiate over how the cost $c$ is split between them, so that a link is formed whenever they jointly benefit from it. It is assumed that future terms of trade are noncontractible. 9

In stage two, buyers and sellers bargain over the network. The trades implemented are described by an $m \times n$ matrix $\mathbf{x}$, where element $x_{i j}$ indicates that buyer $i$ purchases $x_{i j} \in[0,1]$ units from seller $j$. All rows and all columns of $\mathbf{x}$ must sum to no more than 1 , as each buyer demands only a single unit and each seller supplies only a single unit. 10

For $x_{i j}>0$, the price buyer $i$ pays seller $j$ per unit is $p_{i j}$. Bargaining solutions that map the potential gains from trade $\mathbf{a}$ and network $L$ into trades $\mathbf{x}(\mathbf{a}, L)$ and prices $\mathbf{p}(\mathbf{a}, L)$ will be considered. Buyer $i$ 's payoff is denoted by $u_{i}(\mathbf{a}, L): \mathbb{R}_{+}^{m \times n}$ $\times 2^{m \times n} \rightarrow \mathbb{R}_{+}$, and seller $j$ 's payoff by $v_{j}(\mathbf{a}, L): \mathbb{R}_{+}^{m \times n} \times 2^{m \times n} \rightarrow \mathbb{R}_{+}$. The utilities of buyer $i$ and seller $j$ are the surpluses they extract from each of their transactions: ${ }^{11}$

$$
u_{i}=\sum_{k \in Q} x_{i k}\left(a_{i k}-p_{i k}\right) \quad v_{j}=\sum_{k \in P} x_{k j} p_{k j}
$$

The symmetry of buyers and sellers means that for all the results found for buyers, there are symmetric results for sellers. Some results will be stated for just buyers or just sellers.

\footnotetext{
${ }^{9}$ This assumption is discussed in Section VID.

${ }^{10}$ While it is possible for $i$ to purchase $x_{i j} \in(0,1)$ of a unit from seller $j$ and $x_{i k}$ of a unit from another seller $k$, it will turn out that endogenously $x_{i j} \in\{0,1\}$.

${ }^{11}$ Without loss of generality, sellers' values from keeping their goods are normalized to zero, and buyers' values of goods are adjusted to reflect the net gains from trade.
} 


\section{Bargaining over a Formed Network}

To analyze network formation, a backward induction approach will be applied and trade over a given network considered first. This section proposes a mapping from the formed network $(L)$ and potential gains from trade (a) into outcomes (quantities traded by different pairs and prices) and identifies how the network structure affects these outcomes.

A bargaining outcome is a triple $(\mathbf{x}, \mathbf{u}, \mathbf{v})$. Bargaining outcomes are considered that satisfy pairwise stability. An outcome $(\mathbf{x}, \mathbf{u}, \mathbf{v})$ is stable if payoffs are individually rational $\left(u_{i} \geq 0, v_{j} \geq 0\right.$ for all $\left.i, j\right)$ and no buyer and seller could both increase their payoffs by trading with each other and splitting the resulting gains from trade in any way: for all $i, j, u_{i}+v_{j} \geq a_{i j}$ if $l_{i j} \in L$. The set of stable outcomes is denoted by $\Omega .12$

Let the matrix $\alpha$, with elements $\alpha_{i j}:=a_{i j} l_{i j}$, summarize the values of feasible trades after investments have been made. Unlinked buyer-seller pairs then generate no surplus if they match, and will do equally well by remaining unmatched. The bargaining over a network problem with potential gains from trade a and possible trades given by the network $L$ is then a matching with transferable utility (assignment) problem with gains from trade $\alpha$.

A match $\mu$ is a function from the set of all buyers and sellers into itself, $\mu: P \cup Q \rightarrow P \cup Q$, such that (i) each buyer (seller) is matched to a seller (buyer) or himself (herself), and (ii) buyer $i$ is matched to seller $j$ if and only if seller $j$ is matched to buyer $i .^{13}$ Although in principle a buyer can multihome and buy from multiple sellers, such outcomes are typically unstable. Generically, ${ }^{14}$ the quantity traded $x_{i j}$ is an element of $\{0,1\}$ in all stable outcomes. This follows from the linearity in the utility functions. It is then without loss of generality that bargaining outcomes consisting of the triple $(\mu, \mathbf{u}, \mathbf{v})$ are considered. Denote the set of all possible matches $M$. Where there should be no confusion, notation will be abused and arguments dropped.

There is a generically unique match $\mu^{*}(\cdot ; \boldsymbol{\alpha})$ that maximizes the gains from trade:

$$
\mu^{*}(\cdot ; \boldsymbol{\alpha}):=\underset{\mu \in M}{\arg \max }\left\{\sum_{i \in P} \alpha_{i \mu(i)}\right\}
$$

Shapley and Shubik (1972) show that the set of stable outcomes (matches and payoffs) has many nice properties. ${ }^{15}$ First, we know that there exist payoffs for which a match is stable if and only if that match maximizes the total surplus. This implies that there is a (generically) unique pairwise stable match. Second, when

\footnotetext{
${ }^{12}$ An alternative approach would be to require bargaining outcomes to satisfy the Shapley value (Myerson 1977). However. in this environment, the Shapley value is not always stable (consider, for example, the network shown ir Figure 2).

${ }^{13}$ Formaliy: $\mu(i ; \boldsymbol{\alpha}) \in Q \cup i ; \mu(j ; \boldsymbol{\alpha}) \in P \cup j$; and $\mu(i ; \boldsymbol{\alpha})=j$ if and only if $\mu(j ; \boldsymbol{\alpha})=i$.

${ }^{14}$ Let $\varepsilon_{i j}$ be a noise term drawn independently from an atomless distribution with continuous support. If we take any gains from trade and perturb them by adding these noise terms, and so consider gains from trade $\hat{a}_{i j}=a_{i j}+\varepsilon_{i j}$, then with probability $1, x_{i j} \in\{0,1\}$ for all $i, j$ in all stable outcomes. See Roth and Sotomayor (1990).

${ }^{15}$ We discuss and provide intuition for these results in Section OA-1 of the online Appendix.
} 
there are multiple surplus-maximizing matches, the same payoffs support all such matches as a stable outcome. Third, each matched buyer and seller must split their gains from trade between themselves in a stable outcome: $u_{i}+v_{\mu^{*}(i)}=\alpha_{i j}$. Fourth, even though we look only for outcomes robust to pairwise deviations, this also implies there are no profitable coalitional deviations- the set of stable outcomes coincides with the set of core outcomes. Finally, the set of stable payoffs forms a complete lattice for the partial ordering of buyers' payoffs, ${ }^{16}$ where $\mathbf{u}>\mathbf{u}^{\prime}$ if and only if $u_{i}>u_{i}^{\prime}$ for all $i \in P$. $^{17}$

It will be helpful to define the lowest and highest stable payoffs the agents can receive:

$$
\begin{array}{llll}
\underline{u}_{i} & :=\min _{(\mu, \mathbf{u}, \mathbf{v}) \in \Omega} u_{i}, & \bar{u}_{i}:=\max _{(\mu, \mathbf{u}, \mathbf{v}) \in \Omega} u_{i}, \\
\underline{v}_{i}:=\min _{(\mu, \mathbf{u}, \mathbf{v}) \in \Omega} v_{i}, & \bar{v}_{i}:=\max _{(\mu, \mathbf{u}, \mathbf{v}) \in \Omega} v_{i} .
\end{array}
$$

We will refer to a buyer $i$ 's lowest possible stable payoff $\left(\underline{u}_{i}\right)$ as his "outside option." Seller $j$ 's outside option $\left(\underline{v}_{j}\right)$ is defined analogously. The lattice structure of the stable outcomes implies that the payoff vectors $(\underline{\mathbf{u}}, \overline{\mathbf{v}})$ and $(\overline{\mathbf{u}}, \underline{\mathbf{v}})$ are stable.

Figure 2 shows a simple formed network. On this network, $b_{1}$ will be matched to seller $s_{1}$ (otherwise $b_{1}$ and $s_{1}$ would have a profitable deviation). The worst-case scenario for $b_{1}$ is to receive a payoff of 5 . If $b_{1}$ received any worse terms of trade, he would have a profitable deviation by instead trading with $s_{2}$. Thus, $\underline{u}_{b_{1}}=5$ and the set of stable outcomes are $u_{b_{1}}=5+5 \beta, v_{s_{1}}=5(1-\beta)$ and $v_{s_{2}}=0$ for a parameter $\beta \in[0,1]$.

Demange, Gale, and Sotomayor (1986) identify an algorithm to find buyers' and sellers' minimum stable payoffs. However, not much is known about how the structure of the network $\alpha$ affects payoffs. The network decomposition algorithm identifies a simple way of decomposing any weighted network $\boldsymbol{\alpha}$ into a directed weighted network from which the effect of every link on each agent's outside option can be easily found:18

- Identify trade partners. Given a network $\alpha$, find the match that maximizes the possible gains from trade. This match $\left(\mu^{*}(\cdot ; \boldsymbol{\alpha})\right)$ defines each agent's trade partner. Represent these relationships by directed solid links.

- Identify outside trade partners. To find an agent $k$ 's outside trade part$n e r$, remove his trade partner $\mu^{*}(k)$ from the network and identify the agent he rematches to for the gains from trade to be maximized over the new network. ${ }^{19}$ More formally, let $\boldsymbol{\alpha} / \mu^{*}(k ; \boldsymbol{\alpha})$ be the gains from trade once player $\mu^{*}(k ; \boldsymbol{\alpha})$ is removed from the network so that $i$ and $j$ have gains from trade $\alpha_{i j}$ if $i, j \neq \mu^{*}(k ; \boldsymbol{\alpha})$, and 0 otherwise. Agent $k$ 's outside trade partner is then

\footnotetext{
${ }^{16}$ The equivalent partial ordering for sellers' payoffs could be used.

${ }^{17}$ More formally, suppose $(\mathbf{u}, \mathbf{v})$ and $\left(\mathbf{u}^{\prime}, \mathbf{v}^{\prime}\right)$ are stable payoff vectors. Let $\hat{\mathbf{u}}=\mathbf{u} \vee \mathbf{u}^{\prime}$, such that for all $i, \hat{u}_{i}=\max \left(u_{i}, u_{i}^{\prime}\right) ;$ and let $\tilde{\mathbf{v}}=\mathbf{v} \wedge \mathbf{v}^{\prime}$, such that for all $j, \tilde{v}_{j}=\min \left(v_{j}, v_{j}^{\prime}\right)$. Define $\tilde{\mathbf{u}}$ and $\hat{\mathbf{v}}$ analogously. The payoff vectors $(\hat{\mathbf{u}}, \tilde{\mathbf{v}})$ and $(\tilde{\mathbf{u}}, \hat{\mathbf{v}})$ must then also be stable.

${ }^{18}$ Generically, there is a unique network decomposition. When there are multiple directed network representations, any one can be selected and all results will carry through.

${ }^{19}$ If $k$ is unmatched, $k$ has no outside trade partner.
} 


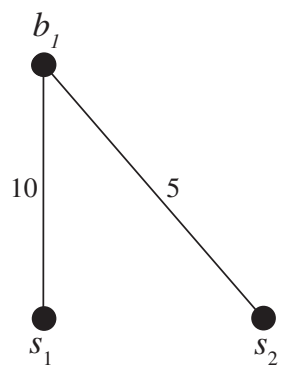

Figure 2. Simple NeTWORK

Panel A. Formed network

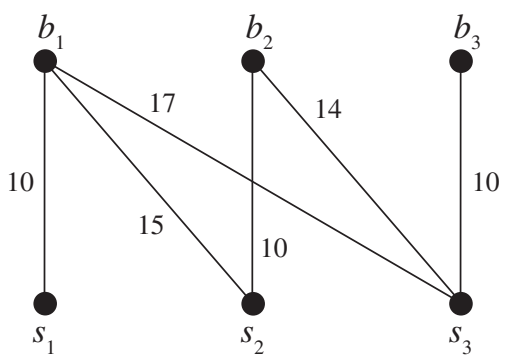

Panel B. Decomposition

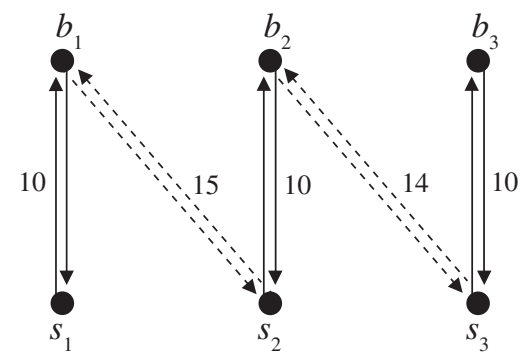

Figure 3. Directed Network Decomposition

$\eta(k ; \boldsymbol{\alpha}):=\mu^{*}\left(k ; \boldsymbol{\alpha} / \mu^{*}(k ; \boldsymbol{\alpha})\right)$. Find all agents' outside trade partners in this manner, and represent these relationships (outside option links) by directed dashed links from $k$ to $\eta(k)$.

The network decomposition algorithm runs in polynomial time and can be programmed as the solution to $m+n+1$ linear programming problems. To see how the decomposition is implemented (by hand), consider the network in Figure 3, panel A. The links traded over are found first by considering the match that maximizes the gains from trade. These relationships are represented by directed solid links in Figure 3, panel B. For example, $b_{1}$ must trade with $s_{1}$ for the gains from trade to be maximized, and so $s_{1}$ is $b_{1}$ 's trade partner. To find each agent's outside trade partner, his trade partner is removed from the network and the match that maximizes the gains from trade on this reduced network is found. If $s_{1}$ is removed from the network, $b_{1}$ has to rematch to $s_{2}$ for the gains from trade to be maximized, and so $b_{1}$ 's outside trade partner is $s_{2}$, as shown by the dashed directed link from $b_{1}$ to $s_{2}$. It is interesting that in this example the highest-value link does not feature in the decomposed network and so is redundant.

Agent $k$ 's outside option chain is a sequence of links that can be easily identified from the network decomposition. Start at the node $k$. Then alternately follow the directed dashed links and then directed solid links, until this is no longer possible. Let $L_{k}^{d l}$ denote the set of dashed links in $k$ 's outside option chain, and $L_{k}^{s l}$ the set of 
solid links in $k$ 's outside option chain. ${ }^{20}$ The outside option chains for the network in Figure 3 are shown in Figure 5, panel A.

We now show how agents' outside option chains determine the value of their outside options. Suppose $k$ 's trade partner $\left(\mu^{*}(k)\right)$ is removed from the network. The gains from trade being generated by $k$ and $\mu^{*}(k), \alpha_{k \mu^{*}(k)}$, will then be lost, but a rematching may recover some of these losses. Lemma 1 shows that the optimal rematching, which maximizes the gains from trade generated on the network without $\mu^{*}(k)$, occurs along $k$ 's outside option chain: $k$ rematches to his outside trade partner $\eta(k)$, which leaves $\eta(k)$ 's trade partner unmatched, so he rematches to his outside trade partner, and so on. Whenever an agent is rematched in any optimal rematching, he rematches to the same person.

Denote the set of links that are no longer matched over in an optimal rematching following the removal of $\mu^{*}(k)$ by $L_{\mu^{*}(k)}^{-}$, and the set of links that are newly matched over by $L_{\mu^{*}(k)}^{+}$, and recall that the links in $L_{k}^{d l}$ are the dashed links in $k$ 's outside option chain, while the links in $L_{k}^{s l}$ are the solid links in k's outside option chain.

\section{LEMMA 1:}

(i) If an agent is removed from the network, every agent displaced as part of the subsequent optimal rematching is rematched to his outside trade partner-the same agent he would rematch to if his trade partner were directly removed from the network:

$$
L_{\mu^{*}(k)}^{+}=L_{k}^{d l} \quad \text { and } \quad L_{\mu^{*}(k)}^{-}=L_{k}^{s l} \cup\left\{l_{k \mu^{*}(k)}\right\} \quad k \in P \cup Q .
$$

(ii) Outside option chains are always finite; they never cycle.

The Proof of Lemma 1 and subsequent proofs are in the Appendix, section B. To gain intuition for Lemma 1, consider the simple three-buyer and three-seller network shown in Figure 4. The dashed ovals represent the optimal match on this network. Now suppose that $s_{1}$ is removed from the network and in the optimal rematching $b_{2}$ rematches to $s_{3}$, as shown in Figure 4. One implication of Lemma 1 is that $b_{2}$ must also rematch to $s_{3}$ if $s_{2}$ were removed from the network instead of $s_{1}$; thus the rematching shown in Figure 4, panel C cannot be optimal.

To see why the rematchings in Figure 4 are inconsistent, note that the following two equations must hold:

$$
\begin{gathered}
\alpha_{11}+\alpha_{22}+\alpha_{33}>\alpha_{12}+\alpha_{21}+\alpha_{33}, \\
\alpha_{12}+\alpha_{23}>\alpha_{22}+\alpha_{33} .
\end{gathered}
$$

\footnotetext{
${ }^{20}$ Outside option chains, although defined and motivated in a different way, are similar to the opportunity paths identified by Kranton and Minehart (2000a) for networks with homogeneous gains from trade. Outside option chains can be viewed as a generalization of opportunity paths. However, there is no counterpart to Theorem 1 in Kranton and Minehart (2000a). In concurrent independent work, Caplin and Leahy (2014, 2010) have identified similar mathematical structures using a different methodology.
} 
Panel A. Formed network

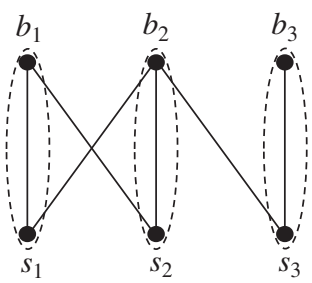

Panel B. Removing $s_{1}$

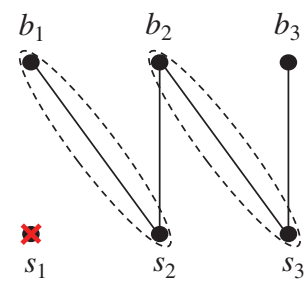

Panel C. Removing $s_{2}$

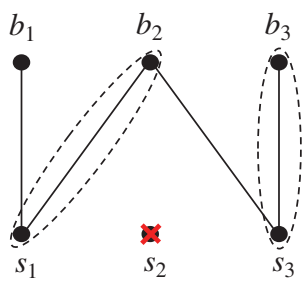

Figure 4. Inconsistent Optimal Rematchings which Violate Lemma 1

Equation 2 holds from the optimal match being the one shown in Figure 4, panel A; and Equation 3 holds from the optimal match shown in Figure 4, panel B. Summing these inequalities:

$$
\alpha_{11}+\alpha_{23}>\alpha_{21}+\alpha_{33}
$$

It follows immediately that the rematching shown in Figure 4, panel $\mathrm{C}$ does not maximize the gains from trade and cannot be optimal. The Proof of Lemma 1 generalizes this intuition, showing that on any finite network no players rematch to anyone other than their outside trade partner. Lemma 1 provides the key step in proving Theorem 1, which shows how agents' outside options can be found from the directed network decomposition.

THEOREM 1: Each agent's outside option can be found by alternatively adding and then subtracting the values of the links in his outside option chain:

$$
\underline{u}_{i}=\sum_{l \in L_{i}^{d l}} \alpha_{l}-\sum_{l \in L_{i}^{s l}} \alpha_{l}, \quad \quad \underline{v}_{j}=\sum_{l \in L_{j}^{d l}} \alpha_{l}-\sum_{l \in L_{j}^{s l}} \alpha_{l}
$$

Whenever an agent's trade partner $\left(\mu^{*}(i)\right)$ is removed from the network, by Lemma 1 the optimal rematching occurs along $i$ 's outside option chain. The change in total surplus after $\mu^{*}(i)$ is removed can be found by adding the new links matched over and subtracting the old links no longer matched over. This change in total surplus is $\mu^{*}(i)$ 's marginal contribution to total surplus. Using a result from Démange (1982) and Leonard (1983), this is also $\mu^{*}(i)$ 's maximum possible pairwise stable payoff. As $i$ and $\mu^{*}(i)$ must split the surplus they generate, and by the lattice structure of the pairwise stable payoffs, $\alpha_{i \mu^{*}(i)}$ less $\mu^{*}(i)$ 's marginal contribution to total surplus is $i$ 's outside option.

Outside option chains for the network given in Figure 3 are illustrated in Figure 5, panel A. Each agent's outside option is then found by alternately adding and subtracting the values of these links. For example, $b_{1}$ 's outside option chain consists of the sequence of links $\left\{l_{b_{1} s_{2}}, l_{b_{2} s_{2}}, l_{b_{2} s_{3}}, l_{b_{3} s_{3}}\right\}$ and so his outside option is given by $\alpha_{b_{1} s_{2}}-\alpha_{b_{2} s_{2}}+\alpha_{b_{2} s_{3}}-\alpha_{b_{3} s_{3}}$, as shown in Figure 5, panel B. 
Panel A. Outside option chains

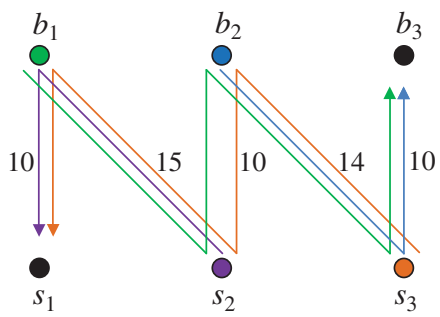

Panel B. Outside options

\begin{tabular}{c|l} 
Agent & Outside option \\
\hline$b_{1}$ & $15-10+14-10=9$ \\
$b_{2}$ & $14-10=4$ \\
$b_{3}$ & 0 \\
$s_{I}$ & 0 \\
$s_{2}$ & $15-10=5$ \\
$s_{3}$ & $14-10+15-10=9$
\end{tabular}

Figure 5. Directed Network Decomposition

There is a strong relationship between the outside trade partners identified in the network decomposition algorithm and players' profitable pairwise deviations. ${ }^{21}$

COROLLARY 1: Consider any stable outcome. Suppose agent k's terms of trade are now weakened to the extent that he receives a payoff below his outside option. Agent $k$ will then have a profitable deviation by trading with his outside trade partner $\eta(k)$.

Corollary 1 shows that it is the potential for an agent to trade with his outside trade partner that generates a binding constraint on his minimum possible stable payoff and establishes his outside option.

Stability implies that for all buyers $i, u_{i} \in\left[\underline{u}_{i}, \alpha_{i \mu^{*}(i ; \boldsymbol{\alpha})}-\underline{v}_{\mu^{*}(i ; \boldsymbol{\alpha})}\right]$, with analogous bounds on sellers' payoffs. ${ }^{22}$ The value of the network decomposition and Theorem 1 is that it identifies how the structure of the network affects agents' outside options and informs us about their incentives to invest in specific links. In the next section we will analyze these investment incentives and show that inefficiencies in investments can be large. Without any further restrictions on the mapping from formed networks to bargained outcomes, such a result would not be too surprising - a mapping from formed networks to stable payoffs could be picked to create bad incentives to form links. To demonstrate the possibility of inefficiencies more forcefully, we will tie our hands and look at point predictions rather than set-valued predictions. Pairwise stability ensures that agents receive at least their outside option from a trade, but their investment incentives will depend crucially on how the remaining surplus is split. To capture this, we generalize the parametrization of stable outcomes found for the network in Figure 2.

Suppose that $i$ is matched to $j$ on the network $\boldsymbol{\alpha}: \mu^{*}(i ; \boldsymbol{\alpha})=j$. Consider terms of trade in which $i$ and $j$ receive their outside options for sure, and then the remaining

\footnotetext{
${ }^{21}$ Recall that outside trade partners are identified by removing an agent's trade partners from the network and seeing who he rematches to. If instead an agent's link to his trade partner is removed from the network and the subsequent rematching is used to identify outside trade partners, it is possible to construct examples in which an agent has an outside trade partner but can still receive a payoff of zero without having a profitable pairwise deviation. See Section OA-4 of the online Appendix.

${ }^{22}$ In the example of Figure 3, panel A, $u_{b 1} \in[9,10], u_{b 2} \in[4,5], u_{b 3} \in[0,1], v_{s 1} \in[0,1], v_{s 2} \in[5,6]$, and $v_{s 3} \in[9,10]$.
} 
gains from trade are split according to an exogenous parameter $\beta \in[0,1]$, which can be interpreted as buyers' relative bargaining power:

$$
\begin{aligned}
& u_{i}=\underline{u}_{i}+\beta\left(\alpha_{i j}-\underline{u}_{i}-\underline{v}_{j}\right), \\
& v_{j}=\underline{v}_{j}+(1-\beta)\left(\alpha_{i j}-\underline{u}_{i}-\underline{v}_{j}\right) .
\end{aligned}
$$

Focusing on these terms of trade, with an exogenously specified bargaining-power parameter, emphasizes the role of the network in providing outside options. In Corominas-Bosch (2004), players are selected at random and then make offers simultaneously to all of their possible trade partners. The terms of trade in equations (5)and (6) are the same as those found in Corominas-Bosch (2004), although she analyzes only environments with homogeneous gains from trade. ${ }^{23}$

As in all stable payoffs in which $i$ and $j$ are matched $u_{i}+v_{j}=\alpha_{i j}$, we have that $\bar{u}_{i}+\underline{v}_{j}=\underline{u}_{i}+\bar{v}_{j}=\alpha_{i j}$. Equations (5) and (6) can therefore be rewritten as:

$$
\begin{aligned}
& u_{i}=\beta \bar{u}_{i}+(1-\beta) \underline{u}_{i}, \\
& v_{j}=(1-\beta) \bar{v}_{j}+\beta \underline{v}_{j} .
\end{aligned}
$$

These payoffs are a convex combination of agents' maximum and minimum possible stable payoffs. When $\beta=1$, all buyers receive their maximum possible payoffs, while sellers receive their minimum possible payoffs; and when $\beta=0$, all buyers receive their minimum possible payoffs, while sellers receive their maximum possible payoffs. At both these payoff vectors, no buyer $i$ and seller $j$ has a profitable pairwise deviation, so $\bar{u}_{i}+\underline{v}_{j} \geq \alpha_{i j}$ and $\underline{u}_{i}+\bar{v}_{j} \geq \alpha_{i j}$. It follows immediately that for any $\beta \in[0,1], \beta\left(\bar{u}_{i}+\underline{v}_{j}\right)+(1-\beta)\left(\underline{u}_{i}+\bar{v}_{j}\right) \geq \alpha_{i j}$, so the payoffs from equations (5) and (6) are stable. ${ }^{24}$

With the above parametrization of agents' payoffs, the network decomposition algorithm identifies who has incentives to form which links. It is straightforward to substitute the result from Theorem 1 into the agents' payoff functions (equations (5) and (6)) to find how the value of each link affects each agent's payoff at the margin. This will be very helpful when considering the network formation problem in Section V. For example, if a link would be redundant, there will be no incentives to invest in it; despite $l_{b_{1} s_{3}}$ being the highest-value link in Figure 3, panel A, neither $b_{1}$ nor $s_{3}$ will be willing to invest in it if the other links are formed.

\footnotetext{
${ }^{23}$ It is not straightforward to directly extend Corominas-Bosch's model to include heterogeneous gains from trade. Indeed, she concludes that although it is "natural to ask for the introduction of a little bit of heterogeneity in the model $[\ldots][\mathrm{w}] \mathrm{e}$ believe that this line of research is unlikely to lead to fruitful results." Section OA-3 of the online Appendix shows the equivalence of the Corominas-Bosch (2004) outcomes and the terms of trade in equations 5 and 6.

${ }^{24}$ These terms of trade are generally only a subset of the stable terms of trade. Were individualized pairwise bargaining powers $\beta_{i j}$ modeled, all pairwise outcomes would be included but also some nonstable outcomes. For a complete characterization of pairwise stable outcomes see Quint (1991).
} 


\section{Efficiency Losses}

Using the simple mapping from formed networked markets into bargaining outcomes provided by combining Theorem 1 with equations (5) and (6), investment inefficiencies in the network formation stage will be analyzed. Ideally, statements would be made about how efficiency losses change with the potential gains from trade between different buyers and sellers, the relative bargaining power of buyers to that of sellers, and how the costs of forming links is split. However, a number of difficulties inhibit such statements. There are typically multiple stable networks, and so comparisons across sets of networks are required. Moreover, the set of stable networks does not have a simple mathematical structure that could facilitate set-based statements. ${ }^{25}$ And even when there is a unique stable network, efficiency losses can be nonmonotonic in the model's parameters. ${ }^{26}$ Despite these problems, some progress will be possible by identifying upper and lower bounds on inefficiency that apply to all stable networks.

\section{A. The Efficient Network}

The efficient network maximizes the net gains from trade $(N G T(L))$ - the gains generated from trade less the costs of forming the links in $L$ :

$$
L^{e}:=\max _{L \in \mathcal{L}} N G T(L)=\max _{L \in \mathcal{L}}\left\{\sum_{i \in P} \alpha_{i \mu^{*}(i, \boldsymbol{\alpha}(L))}-c|L|\right\},
$$

where $\alpha_{i i}=0$ for each buyer $i \in P ;|L|$ is the cardinality of $L$ and so the number of links in network $L$; and $\mathcal{L}$ is the set of all possible bipartite networks.

\section{B. Measuring Inefficiencies}

The efficiency loss $E L(L)$ of a network $L$ will be measured as the percentage of the possible net gains from trade that are lost: $E L=\left(N G T\left(L^{e}\right)-\right.$ $N G T(L)) / N G T\left(L^{e}\right) \in[0,1]$. There are then two subtleties to deal with. First, when the supremum of possible efficiency losses for all potential gains from trade is 1 , it will be said that the efficiency losses are 100 percent. Second, when there are multiple stable networks, a conservative approach will be taken, and only results that apply to all stable networks will be stated. ${ }^{27}$

\footnotetext{
${ }^{25}$ More specifically, the set of stable networks does not form a complete lattice or semi-lattice when partially ordered by set inclusion of links, inhibiting comparisons that might otherwise be made in the strong set order.

${ }^{26}$ See Section OA-9 statics of the online Appendix.

${ }^{27}$ Equivalently, we use the "price of anarchy" and "cost of anarchy" (Koutsoupias and Papadimitriou 2009; Roughgarden and Tardos 2004), by considering the worst stable network when bounding the efficiency losses from above and the best stable network when bounding inefficiency losses from below.
} 


\section{Types of Inefficiency}

Overinvestment inefficiency $O I I(L)$ on a network $L$ is the efficiency loss due to the formation of links that are not used for trade. Denoting the links in a network $L$ used for trade by $K \subseteq L$, the efficiency loss due to overinvestment in non-trade links is ${ }^{28}$

$$
O I I(L):=\frac{(|L|-|K|) c}{N G T(L)+(|L|-|K|) c} \in[0,1] .
$$

Overinvestment inefficiency occurs when a link that is not used for trade is formed. An agent might want to form such a link to establish an outside option and affect his terms of trade with his trade partner.

Underinvestment inefficiency $(U I I(L))$ on a network $L$ is the efficiency loss due to forgone net gains from trade that could be realized by forming links between unmatched buyers and unmatched sellers. ${ }^{29}$ Consider a network $L$, such that buyers $\hat{P} \subseteq P$ and sellers $\hat{Q} \subseteq Q$ will end up unmatched in stable outcomes. For this subset of buyers and sellers, denote the efficient network by $\hat{L}^{e}:=\max _{L^{\prime} \in \hat{\mathcal{L}}} N G T\left(L^{\prime}\right)$, where $\hat{\mathcal{L}}$ is the set of all possible bipartite networks between the buyers $\hat{P}$ and sellers $\hat{Q}$. Underinvestment inefficiency on $L$ is then ${ }^{30}$

$$
U I I(L):=\frac{N G T\left(\hat{L}^{e}\right)}{N G T(L)+N G T\left(\hat{L}^{e}\right)} \in[0,1] .
$$

Underinvestment inefficiency can occur due to a hold-up problem. Unmatched buyers and sellers may have positive net gains from trade but remain unlinked on a stable network, anticipating that they will be held up once they have sunk their investment costs. ${ }^{31}$

\section{Inefficiencies in Stable Networks}

In this section, we compare stable (equilibrium) networks to the efficient network.

\section{A. Protocol A}

We first assume that cost shares are exogenous, so that buyers and sellers must make separate, nonsubstitutable investments to enable trade. For a link to be formed, the buyer must pay $\gamma c$, while the seller pays $(1-\gamma) c$, where $c>0$ and $\gamma \in[0,1]$.

The simultaneous link formation game is a simultaneous move game with complete information. Buyers and sellers are the players. Each player chooses a subset

\footnotetext{
${ }^{28}$ All results go through, with the same proofs, if the denominator in equation 10 is changed to $N G T\left(L^{e}\right)$.

${ }^{29}$ While it may also be possible to increase the net gains from trade by forming links involving either a matched buyer and/or a matched seller, this will necessarily result in a link currently matched over becoming redundant. Inefficiencies of this type are attributed to coordination problems.

${ }^{30}$ All results go through, with the same proofs, if the denominator in equation 11 is changed to $\operatorname{NGT}\left(L^{e}\right)$.

${ }^{31}$ There may be positive levels of inefficiency remaining once overinvestment and underinvestment inefficiency have been taken into account. This inefficiency can be attributed to coordination problems.
} 
of the players on the other side of the market to attempt to form a link to. Buyers invest $\gamma c$ in each link they select, while sellers invest $(1-\gamma) c$ in each link they select. A buyer $i \in P$ therefore has a strategy set $\{0,1\}^{n}$, while a seller $j \in Q$ has a strategy set $\{0,1\}^{m}$. A link is formed if and only if a total investment of $c$ is made in it. Links therefore require investment from both the buyer and seller to be formed, except when $\gamma \in\{0,1\}$. Payoffs are players' payoffs from trade on the formed network less their investment costs.

If stable networks were defined to be a pure strategy Nash equilibrium of the simultaneous link formation game, there would be stable networks where a buyer and a seller fail to coordinate on forming a link that would benefit them both. Furthermore, the empty network would be stable for all $\gamma \in(0,1)$. To eliminate these types of networks from the set of stable networks, buyers and sellers will be allowed to coordinate on forming a link that benefits them both.

A network will be considered stable when it is pairwise Nash stable. A network $L$ is pairwise Nash stable ${ }^{32}$ if and only if it is a pure strategy Nash equilibrium of the simultaneous link formation game and pairwise stable, so that no unformed link would benefit both the buyer and seller were it formed. Formally, pairwise stability requires that, for all $i, j$, such that $l_{i j} \notin L$, the following conditions hold: ${ }^{33}$

- If $u_{i}\left(L \cup l_{i j}\right)-\gamma c>u_{i}(L)$, then $v_{j}\left(L \cup l_{i j}\right)-(1-\gamma) c<v_{j}(L)$.

- If $v_{j}\left(L \cup l_{i j}\right)-(1-\gamma) c>v_{j}(L)$, then $u_{i}\left(L \cup l_{i j}\right)-\gamma c<u_{i}(L)$.

Unlike pairwise stability alone, pairwise Nash stability ensures that agents' "individual rationality" constraints are met because agents always have the option of deleting all their links and receiving a payoff of zero. Networks that are pairwise stable, but not a Nash equilibrium of the simultaneous link formation game, can involve agents receiving negative payoffs without having a profitable deviation because the only deviations they are able to consider are those that delete or add one link at a time. Although stronger than pairwise stability, pairwise Nash stability is a relatively weak stability criterion. A network can be pairwise Nash stable even if a buyer and a seller could profit from forming a link between themselves while simultaneously deleting some other links. However, as discussed below, our results that use pairwise Nash stability are robust to other, stronger stability requirements.

PROPOSITION 1: Under investment protocol A, the following hold:

(i) For all gains from trade $\mathbf{a}$, all cost shares $\gamma \in(0,1)$, and all levels of buyer bargaining power $\beta \in[0,1]$, overinvestment inefficiency on all stable

\footnotetext{
${ }^{32}$ Jackson and Wolinsky (1996) introduced the concept of pairwise stability and suggested pairwise Nash stability as a refinement.

${ }^{33}$ For all potential gains from trade $(\mathbf{a})$, for all levels of bargaining power $(\beta)$, and for cost shares $\gamma \in(0,1)$ there exists a pairwise Nash stable network where all inefficiency can be attributed to underinvestment and overinvestment. This helps motivate the focus on underinvestment and overinvestment inefficiency rather than coordination problems. See Section OA-5 of the online Appendix.
} 
networks is bounded by a function $h(\gamma, \beta, m, n) \in[0 \%, 50 \%]$, and this bound is tight.

(ii) For all nonempty sets $P, Q$ of buyers and sellers, respectively, and all $\beta \neq \gamma$, there exist gains from trade a with underinvestment inefficiency of 100 percent on all stable networks.

Proposition 1 is proved in the Appendix, section B. The second part is proved by constructing an example in which there is a unique stable network that has 100 percent efficiency loss for any $\beta \neq \gamma$ and any $m, n \geq 1$. The proof of the first part of Proposition 1 is more involved and relies heavily on the network decomposition algorithm. Without restricting the possible gains from trade a, it is possible to place an upper bound (which is at most 50 percent) on overinvestment inefficiency in the worst stable network. The upper bound of 50 percent is obtained only for $\beta=\gamma=\frac{1}{2}$. For all other parameter values, the overinvestment bound is tighter. Indeed, as all the bargaining power is concentrated at either the buyers $(\beta \rightarrow 1)$ or the sellers $(\beta \rightarrow 0)$, the upper bound on overinvestment inefficiency goes to zero. To illustrate this, the function $h(\gamma, \beta, m, n)$ is shown for $m=n=10$ in Figure 6. There are similar bounds for other values of $m$ and $n$.

To gain intuition for the efficiency bound in Figure 6, consider the incentives of a matched buyer and matched seller to form an outside option link with each other. For interior cost shares $(\gamma \in(0,1))$, both the buyer and seller must invest in the outside option link for it to be formed. When the buyer's bargaining power is high ( $\beta$ high), he has little incentive to make this investment; the buyer already receives most of the surplus an outside option would guarantee him. The outside option link will not be formed if the buyer has high bargaining power and has to pay at least some moderate cost toward forming the link. Similarly, the seller will not be willing to invest much in an outside option when his bargaining power is high ( $\beta$ low). This explains why the inefficiency bound goes to 0 as $\beta \rightarrow 0$ and as $\beta \rightarrow 1$.

We now provide some intuition for why the bound is never more than 50 percent. Consider a matched buyer-seller pair $(i, j)$, where both the buyer $i$ and seller $j$ have no other links, and consider the maximum amount that $i$ and $j$ would each be willing to invest in an outside option. For $i$ and $j$ to still trade once they have invested in their outside options, the combined value of their outside options must be weakly less than their gains from trade $\left(\alpha_{i j}\right)$. We will consider the overinvestment possible when both $i$ and $j$ invest in an outside option. Suppose then that both $i$ and $j$ have the opportunity to form an outside option link that will guarantee each of them half of the gains from trade. As between them $i$ and $j$ were already splitting the surplus, the creation of these two outside options can lead to a reallocation of at most half the surplus, and so overinvestment cannot exceed 50 percent. ${ }^{34}$

\footnotetext{
${ }^{34}$ Buyer $i$ 's payoff without his outside option is $\beta\left(\alpha_{i j}-\underline{v}_{j}\right)$, and his payoff with his outside option is $\frac{\alpha_{i j}}{2}+\beta\left(\alpha_{i j}-\frac{\alpha_{i j}}{2}-\underline{v}_{j}\right)$. Thus, $i$ is willing to pay at most $(1-\beta) \frac{\alpha_{i j}}{2}$ for his outside option (an amount independent of $\underline{v}_{j}$ ). A similar calculation reveals that $j$ is willing to pay at most $\beta \frac{\alpha_{i j}}{2}$. Summing these amounts, overinvestment can be at most $\frac{\alpha_{i j}}{2}$.
} 


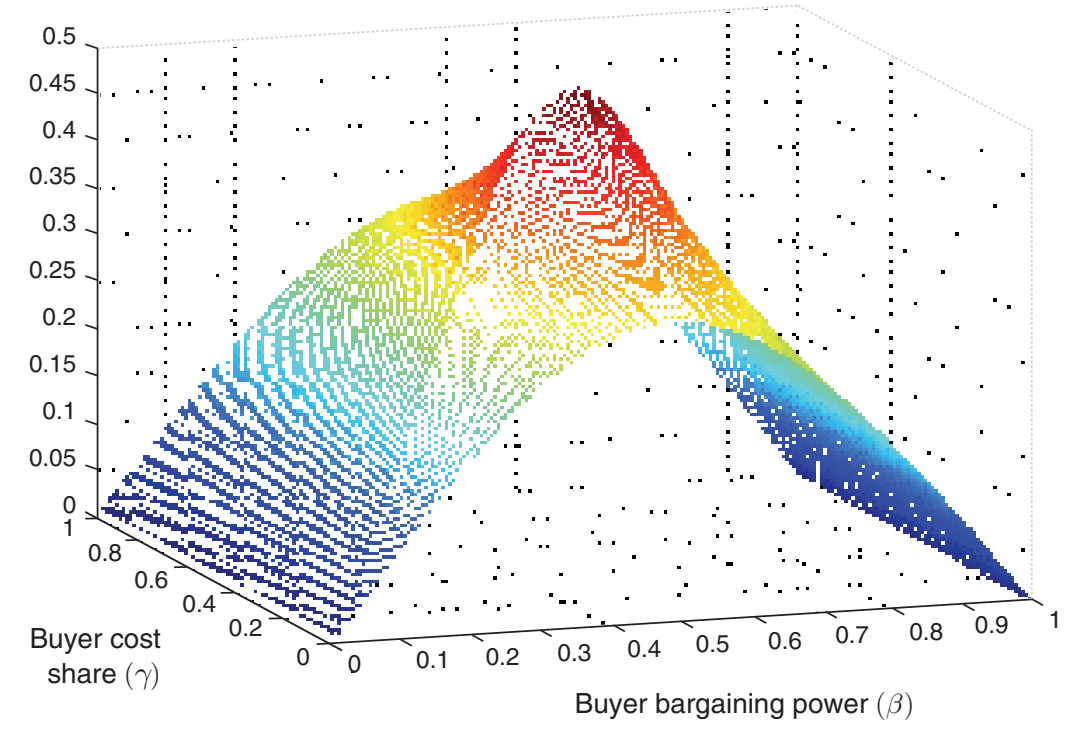

Figure 6. Overinvestment InefFiciency Bound for $m=n=10, \gamma \in(0,1)$, AND $\beta \in(0,1)$

Stability concepts can be categorized by the deviations that they permit. Pairwise Nash stability permits two types of deviations we view as desirable: (i) An agent can adjust his investment in any set of links, ensuring that agents can always delete all their links, thereby guaranteeing that they receive weakly positive payoffs on stable networks, and (ii) any pair of agents can form a new link that would benefit them both, preventing the empty network from being stable. However, there are many stronger definitions of stability that might be used and would permit additional deviations.

Permitting additional deviations can only make fewer networks stable and strengthen the bound on overinvestment inefficiency. Moreover, the constructive proof that 100 percent underinvestment is possible whenever $\beta \neq \gamma$ is robust to permitting many more deviations. As long as agents' strategy sets remain the set of links they can invest in (so that transfers are still not permitted and the mapping from formed networks to payoffs cannot be manipulated), there are no profitable coalitional deviations. More precisely, for the constructive proof in which the empty network is the unique stable network, the empty network is also the unique strong Nash equilibrium of the simultaneous link formation game. ${ }^{35}$ Proposition 1 therefore extends to several stronger definitions of stability.

\footnotetext{
${ }^{35}$ A strong Nash equilibrium (Aumann 1959) requires every coalition to play a best response. For a formal definition, and a proof of the statement, see Corollary 5 in the Appendix, section B.
} 


\section{B. Protocol B}

A critical assumption underlying Proposition 1 is that buyers and sellers cannot negotiate their investment shares. Underinvestment occurs when a buyer and a seller have positive net gains from trade by forming a link, but either the buyer or seller would extract more than these net gains from trade, thereby leaving the other worse off. If buyers and sellers could negotiate how much they each contribute toward forming a link, they would be able to find a split of the investment costs for which they are both better off. Underinvestment would then be eliminated. This intuition is now made precise.

Suppose now that a link is formed whenever the sum of the increases in a buyer and a seller's utilities is greater than the cost of forming the link. This assumption can be motivated in a couple of ways. Most simply, buyers and sellers just have to make a joint investment where investment by one of them is perfectly substitutable with investment by the other. Alternatively, buyers and sellers may be able to make transfers to one another based on the (nonsubstitutable) investments they make.

To accommodate negotiated cost shares, we adjust the definition of a stable network. First, the strategy space of the simultaneous link formation game is expanded. Each agent $k$ can now invest $I_{k k^{\prime}} \in[0, c]$ in a link to each potential trade partner $k^{\prime}$. A buyer's strategy space is $[0, c]^{n}$, while a seller's strategy space is $[0, c]^{m}$. Again, a link is formed if and only if a joint investment of at least $c$ is made in it. A network is a Nash equilibrium of the expanded simultaneous link formation game if all agents' investments are mutual best responses. As in all pure strategy Nash equilibria joint investments of either zero or $c$ must be made in each link, agents' strategy spaces can be restricted, without loss of generality, to choosing investment shares $\left(\gamma_{i j}, \gamma_{j i}\right)$, such that $\gamma_{i j}+\gamma_{j i} \in\{0,1\}$.

A network $L$ is pairwise Nash stable with transfers ${ }^{36}$ if and only if it is a pure strategy Nash equilibrium of the expanded simultaneous link formation game and unformed links would not jointly benefit the unconnected agents: $u_{i}(L)+v_{j}(L) \geq$ $u_{i}\left(L \cup\left\{l_{i j}\right\}\right)+v_{j}\left(L \cup\left\{l_{i j}\right\}\right)-c$ for all $l_{i j} \notin L$. The Nash equilibrium requirement again ensures that agents' individual rationality constraints are satisfied, while pairwise stability again prevents the empty network from always being stable.

It is assumed that if an additional link $l^{\prime} \notin L$ were added to $L$, the cost shares over each link $l \in L$ would remain the same on the network $L \cup l^{\prime}$ as they were on $L .{ }^{37}$ Necessary and sufficient conditions for the efficient network to be pairwise Nash stable with transfers are identified and discussed in the online Appendix. ${ }^{38}$ Of particular interest is that a sufficient condition for the efficient network to be stable

\footnotetext{
${ }^{36}$ This terminology is from Bloch and Jackson (2006). The definition we use differs slightly because investments (transfers) are (implicitly) restricted to be weakly less than the cost of link formation $(c)$, although this upper bound never binds.

${ }^{37}$ While this condition restricts the scope of possible profitable deviations (i.e., a link cannot be added and the cost shares of different links simultaneously changed), it is in the same spirit as pairwise Nash stability, which considers a network to be stable even if it is profitable for a link to be jointly formed while other links are simultaneously deleted. In this respect, pairwise Nash stability with transfers is a relatively minimal requirement.

${ }^{38}$ See Section OA-6 of the online Appendix.
} 
is that agents have anti-assortative preferences. ${ }^{39}$ For $\beta \in(0,1)$ and $c$ sufficiently small, this condition is also necessary.

PROPOSITION 2: Under investment protocol B, the following hold:

(i) There is no underinvestment inefficiency in any stable network.

(ii) For all levels of buyer bargaining power $\beta \in[0,1]$, and all set of $P, Q$ of $m \geq 2$ buyers and $n \geq 2$ sellers, respectively, there exist gains from trade a with overinvestment inefficiency of 100 percent on all stable networks.

The Proof of Proposition 2 is in the Appendix, section B. Under protocol B, a buyer-seller pair can always agree to set the buyer's investment cost share equal to his bargaining power $\left(\gamma_{i j}=\beta\right)$. Underinvestment inefficiency is then eliminated. In contrast, gains from trade can always be found in which the agents on the side of the market with lower bargaining power unilaterally establish outside option links to someone on the other side of the market who would otherwise be unmatched. Extending the example from Section I, for any parameter values $\beta, m, n$, such that there are at least two buyers and two sellers, these outside options can be made just valuable enough for the side of the market with lower bargaining power to invest to the extent that all net gains from trade are lost. ${ }^{40}$

\section{Discussion of Results}

Propositions 1 and 2 show that extreme inefficiency is possible under both proto$\operatorname{col} \mathrm{A}$ and protocol B, although the protocol does affect what causes it. ${ }^{41}$ Removing underinvestment inefficiency problems by permitting buyers and sellers to endogenously negotiate their investment shares exacerbates overinvestment inefficiency problems insofar as they can no longer be bounded below 100 percent.

It is interesting to contrast Propositions 1 and 2 with the efficiency results from the previous literature. Kranton and Minehart (2001) show that when buyers have all the bargaining power $(\beta=1)$ and have to pay all the costs of link formation $(\gamma=1)$, the efficient network is stable for any potential gains from trade a. ${ }^{42} \mathrm{It}$ is already well known that this result is sensitive to small changes in assumptions (Jackson 2008). However, Propositions 1 and 2 identify something further. There is a discontinuity in the inefficiency bound at this point, so that the possible efficiency

\footnotetext{
${ }^{39}$ This requires that no two buyers' most preferred seller is the same when prices are zero and no two sellers' most preferred buyer is the same when sellers can extract all their potential gains from trade from each buyer.

${ }^{40} \mathrm{~A}$ stronger stability requirement in which any coalition could realize a profitable deviation would sometimes (but not always) reduce overinvestment inefficiency. The key constraint that prevents efficiency from always being achieved when all coalitional deviations are possible is that transfers between two agents are limited by their joint investment costs. For a case in which all gains from trade are still lost to overinvestment even when all coalitional deviations are possible, consider the example in Section I. The assumptions underlying the overinvestment result in Proposition 2 are discussed in Section VID.

${ }^{41}$ Section OA-6 of the online Appendix identifies conditions under which the stable network is efficient, and Section OA-8 shows how these conditions simplify in a vertically differentiated market.

${ }^{42}$ Kranton and Minehart (2001) also have uncertainty in their model.
} 
losses go from being bounded at 0 percent to potentially consuming all the gains from trade.

COROLLARY 2: When buyers have all the bargaining power $(\beta=1)$ and have to pay all the costs of link formation $(\gamma=1)$, the efficient network is stable for any potential gains from trade a. Furthermore, there does not exist a stable network with any inefficiency due to overinvestment in non-trade links or underinvestment in trade links. However, if $\beta<1$ or $\gamma<1$, or if investments are made under protocol $B$, there exist potential gains from trade a, where, on even the most efficient stable network, all the gains from trade are lost to inefficiencies.

\section{Applications}

\section{A. High-Skill Labor Markets}

There is an extensive "directed search" literature that models workers targeting their search toward certain firms. For the most part, this literature models firms posting nonnegotiable wages, and finds that labor market search is efficient in many dimensions. ${ }^{43}$ However, while firms post wages in some markets, in other markets wages are negotiated. Hall and Krueger (2008) survey workers about the wage determination process for their most recent jobs. They find that somewhere between one-third and two-thirds of workers bargain over their wages, and that wage negotiations are more prevalent in high-skill markets.

A couple of recent papers model simultaneous bargaining between workers and firms following application and interview decisions. These papers typically avoid the technical difficulties generated by modeling multilateral bargaining in the resulting networked market. Gautier and Holzner (2013) is an exception. However, they assume that all potential worker-firm pairs generate a surplus of either one or zero. The approach taken in this paper permits search inefficiencies to be explored in a model with heterogeneous gains from trade, which are a prominent feature of highskill labor markets. ${ }^{44}$

Suppose that a firm and worker must invest in an interview before the worker can be employed by that firm. Preparing for and then conducting the interview can be costly for the firm, especially in high-skill labor markets, where time may have to be invested by senior management. Interview preparation can also be costly for workers, but to a first approximation, firms seem likely to bear the majority of these costs. There are limited ways in which firms can pass interview costs onto workers, and investments by the worker into the interview process cannot (effectively) substitute for effort by the firm. We therefore apply investment protocol A to these markets.

How valuable outside options are to agents depends on their bargaining powers.

\footnotetext{
${ }^{43}$ See, for example, Acemoglu and Shimer (1999), where investments can be made prior to matching to increase the available gains from trade.

${ }^{44}$ Elliott (2014) also uses the second-stage model developed here to determine matches and the terms of trade in networked labor markets. The paper investigates the fragility of labor markets in the presence of shocks. Workers and firms pay only entry costs to search, and so investments are not relationship-specific.
} 
Remark 1: When firms (respectively, workers) have all the bargaining power ( $\beta=1$, respectively, $\beta=0$ ), their payoffs are independent of their outside options. Moreover, how sensitive an agent's payoff is to his outside option is decreasing in his bargaining power-whenever there is a unique efficient match, for a firm $i$, $\partial u_{i} / \partial \underline{u}_{i}=1-\beta$; and for a worker $j, \partial v_{j} / \partial \underline{v}_{j}=\beta$.

Casual observation of high-skill labor markets suggests that workers value their outside options more than firms. While it is common for workers to use alternative employment opportunities to improve their terms of employment, it is harder to find examples of firms using their alternative employment opportunities to affect their terms of trade with workers. This is suggestive of firms' having most of the bargaining power, which also accords with the observation that firms tend to make offers to workers more frequently than workers make offers (or counteroffers) to firms. We therefore assume that $\beta$ is high.

So far, we have argued that firm bargaining power $(\beta)$ is typically high, and the firm's cost share when establishing a link $(\gamma)$ is also typically high. From Section VB, we know that when $\beta=\gamma$ there is no underinvestment. While Proposition 1 shows that large inefficiencies are possible for all $\gamma \neq \beta$, the possible gains from trade for which this will happen get smaller as $\beta$ and $\gamma$ get closer.

COROLLARY 3: For any $\gamma \neq \beta$, there is 100 percent underinvestment inefficiency in a stable network if and only if

$$
c<\max _{i, j} a_{i j}<\max \left\{\frac{\gamma}{\beta} c, \frac{1-\gamma}{1-\beta} c\right\} .
$$

For any $\gamma$ and any $\beta$, define $\gamma^{\prime}=\lambda \gamma+(1-\lambda) \beta$, where $\lambda$ is a parameter. Corollary 3 implies that, for any $\lambda \in(0,1)$, if there is 100 percent underinvestment inefficiency in a stable network for a market $\left(\mathbf{a}, \gamma^{\prime}, \beta\right)$, then there is also 100 percent underinvestment inefficiency in a stable network for the market $(\mathbf{a}, \gamma, \beta)$. As $\gamma$ gets further away from $\beta$, the set of potential surpluses for which there is 100 percent inefficiency in a stable network weakly increases in the strong set order. Section A of the Appendix characterizes the underinvestment inefficiency bound for a simple example and illustrates this implication of Corollary 3.

Corollary 3 also shows that the possibility of underinvestment depends on market thickness. Suppose that the potential surplus any worker-firm pair can generate is drawn independently at random before the game begins. The expected number of surplus draws over the threshold $\max \{\gamma c / \beta,(1-\gamma) c /(1-\beta)\}$, is then a constant fraction of the number of potential worker-firm pairs. If, for example, there are $n=m$ workers and firms, the expected number of such draws is proportional to $n^{2}$. In thicker markets, workers and firms will, on average, have opportunities for better matches, reducing the possibility of underinvestment. Underinvestment is more likely in thin markets, although for any fixed level of market thickness, the possibility of underinvestment goes to zero as $\beta$ approaches $\gamma$. 
We now consider overinvestment. Recall that the function $h(\gamma, \beta, m, n)$ provides a tight bound on overinvestment inefficiency for $\gamma \in(0,1)$ and $\beta \in(0,1)$. ${ }^{45}$

COROLLARY 4: For a fixed $\beta \in(0,1)$, it is precisely when $\gamma=\beta$ that overinvestment is most severe: $\beta=\underset{\gamma \in(0,1)}{\arg \max } h(\gamma, \beta, m, n)$.

The Proof of Corollary 4 is in the Appendix, section B, and the result is illustrated in Figure 6. Although Corollary 4 shows that for a fixed $\beta$, overinvestment is potentially worst when $\beta=\gamma$, the bound on overinvestment is still quite tight when $\beta$ is high (and $\gamma=\beta$ ). For example, if $\beta=\gamma=0.9$ and $m=n=20$, then $h(0.9,0.9,20,20)=0.12$ and overinvestment is at most 12 percent of the potential gains from trade.

This analysis suggests that we should not expect to see too much evidence of underinvestment, especially when markets are fairly thick, but we might see some overinvestment. It also provides a reason to exercise caution when considering labor market interventions that would subsidize interview costs.

\section{B. Industry Consolidation}

When one firm wants to take over another, substantial relationship-specific investments are required. Due diligence costs are directly paid to lawyers, fees are paid to investment banks, and senior executives' time is forgone. Potential acquirers are typically not willing to make unconditional offers prior to due diligence, and while offers conditional on successful due diligence are often made, in practice what constitutes successful due diligence is noncontractible. ${ }^{46}$ As such agreements are typically nonbinding and can be renegotiated, to a first approximation the market behaves as if investments in due diligence precede negotiations to determine the terms of an acquisition.

In the case of acquisitions, there is evidence that industries undergo periods of consolidation and many mergers occur in waves clustered by industry. This suggests that acquisition decisions are often made in a market context. ${ }^{47}$ Links are formed by potential acquirers undertaking a costly due diligence process. Target firms also have to agree to this process, and it may be costly for them to do so-revealing private information, particularly to a competitor, can reduce the value of the firm to other potential acquirers. When mergers occur, there is evidence that the network structure affects the acquisition price. Jarrell and Poulsen (1989) find that competition to acquire a target firm redistributes rents to the target from the acquirer, while James and Wier (1987) study bank acquisitions and show that competition matters on both sides of the market. An acquirer's takeover gains are increasing in the number of alternative target firms and decreasing in the number of other potential acquirers.

\footnotetext{
${ }^{45}$ A closed-form expression for $h(\gamma, \beta, m, n)$ is provided in Lemma 2 in the Appendix, section B.

${ }^{46}$ The following is a quote from Howson (2003): "Not surprisingly, therefore, the buyer will want to retain its freedom to renegotiate some of the fundamental issues $[\ldots]$ such as the price. $[\ldots]$ In common law countries $[. .$.$] the$ document is little more than a non-binding agreement to negotiate."

${ }^{47}$ See Mitchell and Mulherin (1996) and Andrade, Mitchell, and Stafford (2001).
} 
Although, on the face of it, investments in due diligence are nonsubstitutable, Officer (2003) reports that almost two-thirds of the merger agreements announced between 1997 and 1999 included a target termination fee clause. These clauses require the target to pay a fixed fee to the acquirer if the taget does not consummate the proposed merger. ${ }^{48}$ Given these termination fees, protocol B is a better fit than protocol A.

Under protocol B, the theory predicts that there will be no underinvestment but that overinvestment can be severe. Indeed, the practice of finding alternative acquirers to get the acquisition price bid up is common. An example from 2006 is when Arcelor found itself the target of a hostile takeover bid by its competitor Mittal Steel. Arcelor searched for a white knight and persuaded Severstal to enter the bidding. Mittal Steel eventually acquired Arcelor with an improved bid, and Arcelor paid Severstal a $\$ 177$ million "fine" for the failure of Severstal's bid (Dealbook 2006). ${ }^{49}$ Brandenburger and Nalebuff (1996) provide another example. Paraphrasing:

By 1989 Craig McCaw had acquired cellular phone licenses covering 50 million potential customers, but he wanted a national network which he could obtain by acquiring LIN Broadcasting Corporation. Although there were many potential acquirers of LIN, McCaw valued LIN much more than any of them. As with any takeover there were substantial costs associated with the due diligence process-advisory fees would have to be paid and managerial time forgone. Aware that they would likely be outbid by McCaw, no other potential acquirers entered the bidding. LIN responded by agreeing to compensate Bell South for bidding, paying them about $\$ 75$ million. Bell South is estimated to have bid up the acquisition price McCaw paid for LIN by about \$1 billion to about \$6.5 billion.

One way in which overinvestment might be limited is by exclusivity agreements between a target and an acquirer. Such agreements can commit the target to not form links with alternative acquirers, thereby avoiding overinvestment. ${ }^{50}$ While exclusive-dealing contracts can have efficiency benefits, the Delaware Supreme Court has ruled against the use of such takeover defenses in Omnicare, Inc. v. NCS Healthcare, Inc. 51

In 2002, NCS Healthcare was insolvent. After Genesis showed interest in an acquisition, NCS agreed to use takeover defenses, including an exclusive-dealing contract, to prevent it from considering alternative offers. In effect, NCS agreed to bind their hands and to not use an alternative acquirer to bid up their price. However, NCS's competitor, Omnicare, did make an alternative bid for NCS (conditional on due diligence). NCS did not fully consider this bid and did not reveal their books to

\footnotetext{
${ }^{48}$ Termination fees can be large. In 2005 Guidant paid a termination fee of \$705 million to Johnson and Johnson after accepting a competing offer.

${ }^{49}$ Congruent with the model, Officer (2003) finds that termination fee use by targets is beneficial to their shareholders.

${ }^{50}$ Shleifer and Vishney (1986) consider how takeover defenses can benefit a target firm's shareholders. Segal and Whinston (2000) and McAfee and Schwartz (1994) both also recognize potential efficiency benefits from exclusive-dealing contracts.

${ }^{51}$ Delaware courts are influential in corporation law, and the precedent set by the case might dissuade the boards of other takeover targets from taking defensive measures in the future, although subsequent cases such as Orman versus Cullen have helped to limit the scope of the Omnicare decision.
} 
Omnicare. Omnicare (and some NCS stockholders) then took NCS to court, claiming that NCS had neglected their fiduciary responsibilities to stockholders. The case, Omnicare, Inc. v. NCS Healthcare, Inc., ended up in the Delaware Supreme Court, which reached a 3:2 majority decision against NCS. This undermines the role defensive measures might otherwise play in preventing future overinvestments. In accordance with this view, the Omnicare, Inc. v. NCS Healthcare, Inc. decision has been critiqued by Chief Justice Veasey. ${ }^{52}$

\section{Gas Pipelines}

When investments are made in the construction of pipelines, there is not a clear delineation between the investments the buying country and the selling country should make. Indeed, both countries need to contribute to the same investment, the construction of a pipeline between them. Moreover, there is direct evidence of negotiations over construction costs. ${ }^{53}$ We therefore apply protocol B.

Our model abstracts from a couple of potentially important features of the natural gas market. First, it is assumed that the network is bipartite, eliminating the scope for intermediation. Second, not all buyers have the same demand and not all suppliers have the same supply. While the model is quite far removed from the natural gas market application, the contention of this section is that it can nevertheless yield some insights.

Under protocol B there should be no underinvestment, but substantial overinvestment is possible. There are many examples of possible overinvestments. Before the pipeline between Turkmenistan and China was built, Turkmenistan relied largely on exporting to Russia. One motive for building the pipeline with China, in accordance with the forces studied in this paper, appears to be so that Turkmenistan could improve its terms of trade with Russia. ${ }^{54}$

Europe relies heavily on gas supplies from Russia. Moreover, it appears that Russia has much of the bargaining power and is able to extract most of the gains from trade. It is politically infeasible for Europe to not import sufficient gas to heat homes in the winter. Thus, buyer bargaining power $\beta$ is likely low, increasing the incentives for Europe to invest in alternative sources of supply (see Remark 1).

The proposed 3,300 km Nabucco pipeline would connect Europe to gas supplies in the Middle East. One reason for the EU's potential investment in Nabucco, estimated to eventually cost $\$ 12.3$ billion (Reuters (Reuters $05 / 29 / 08)$ ), is likely to improve their terms of trade with Russia. ${ }^{55}$ Alternative pipelines that would connect Europe with Middle Eastern gas supplies, such as the Trans Adriatic Pipeline, have also been proposed. While it might be argued that higher import capacity is necessary

\footnotetext{
${ }^{52}$ See Oravetz (2004).

${ }^{53}$ One example is the pipeline between Turkmenistan and China. Negotiations over how to split the construction costs began in April 2006, and in July 2007 the Chinese National Petroleum Company and Turkmengaz reached an agreement.

54 "After a number of high-profile moves in recent months to raise the price of Turkmen natural gas for Russia, Ukraine, and Iran, [ Turkmen president] Niyazov has now signed a deal with China to build an export pipeline to the east that would break Russia's monopoly on export routes for Turkmen gas" (Cutler 2010).

${ }^{55}$ There are likely to be many other important factors also at play.
} 
to meet rising demand in Europe, it would most likely be cheaper to increase the capacity of existing pipelines. In addition, Russia has proposed building additional pipelines, Nord Stream and South Stream, into Europe. 56

\section{Assumptions}

The model relies on a couple of important assumptions. First, it is assumed that buyer-seller pairs cannot write long-term enforceable contracts that specify their future terms of trade. If it were possible to write such contracts, the incentives for overinvestment would be directly removed, and terms of trade that ensure there is no underinvestment could be negotiated. Whether such contracts are used is an application-specific question. When international pipelines are built to enable the trade of natural resources, there is typically no effective court to enforce contracted future terms of trade and changing political climates can undermine agreements made by current leaders. There are several examples of ex post renegotiation (Wälde 2008). Regarding industry consolidation, as already argued, contracts conditional on due diligence are typically unenforceable. In high-skill labor markets there is also little evidence of contracts being signed prior to interviews.

A second important feature of the model is that transfers are restricted. An agent $k$ cannot make any transfers when cost shares are exogenous, and when cost shares are endogenous the transfers $k$ can make are limited to those that compensate other agents for their investment in forming links to $k$. This means that transfers from a buyer $i$ to a seller $j$ cannot be made conditional on the other links $j$ forms, and no transfer is possible between $i$ and $j$ if the link $l_{i j}$ is not formed. Finally, the size of transfers is capped at the cost of link formation $c$. These transfers are sufficient for preventing underinvestment but not overinvestment. If unrestricted transfers from one agent to another could be made contingent on all the links formed by the other agent, the efficient network would always be stable (Bloch and Jackson 2007).

There are mechanisms through which more general transfers than we consider may be possible in practice. In the industry consolidation application, we discussed the potential of exclusivity agreements to make a transfer from $i$ to $j$ conditional on $j$ not forming any other links, thereby reducing overinvestment. Also, in a dynamic version of the model, the threat of overinvestment could be sufficient to affect the terms of trade. The instances of overinvestment discussed in relation to the applications in Section VI suggest that at least some of the time such threats alone are not enough. Finally, in some applications, interactions are dynamic and the costs associated with maintaining links overshadows the costs of establishing links. ${ }^{57}$ In these cases, dynamic game effects are likely to be more important.

\footnotetext{
${ }^{56}$ At present, gas from Russia has to travel through either Ukraine or Belarus, with 80 percent coming through Ukraine, to reach Europe. This has led to hold-up problems and disputes. The planned Nord Stream and South Stream pipelines would transport natural gas directly into Europe.

${ }^{57}$ Lee and Fong (2013) have a dynamic model that takes an approach along these lines.
} 


\section{Conclusions}

In many markets a buyer and a seller must make a relationship-specific investment before they can trade. We study inefficiencies in these markets by building on the work of Kranton and Minehart (2000b, 2000a, 2001). We extend their model to consider different levels of bargaining power, different costs shares, negotiated investments, and ex ante heterogeneous gains from trade. These generalizations are important for fitting the model to different applications: both whether there is scope for intervention to avoid inefficiencies, and what this intervention should look like, depend on this fit. We also ask new questions: how inefficient can these investments be? How can these inefficiencies be categorized? This requires new tools. To reveal agents' incentives to invest in different links, we develop an algorithm that identifies how the structure of a networked market influences payoffs. To analyze the size of inefficiencies, we use the cost of anarchy and price of anarchy from the computer science literature and explore the bounds on inefficiency across all stable networks. To investigate the size of different types of inefficiency, we define measures of overinvestment inefficiency and underinvestment inefficiency by adapting the cost of anarchy and price of anarchy to these specific types of inefficiency. We find that when investments are fixed and made separately, overinvestment is limited but-except in a knife-edge case-underinvestment due to hold-up problems can eliminate all the gains from trade. In contrast, when buyers and sellers can negotiate their investment shares, underinvestment inefficiency is eliminated but overinvestment inefficiency can consume all the gains from trade.

We apply the model to three applications: high-skill labor markets, industry consolidation, and the international trade of natural gas. While the model abstracts from details that could be important in all these markets, it nevertheless provides insights into the frictions present. The model can also help shed light on policy questions. For example, it provides an efficiency rationale for exclusivity agreements in networked markets and a framework for evaluating the potential of subsidizing interview costs to improve the functioning of high-skill labor markets. Although beyond the scope of the current paper, there are also modifications that could be made to tailor the model to additional applications. One limitation of the current approach is the unit demand and unit supply assumption. There are several examples of networked markets where this assumption does not fit well: traders of agricultural produce in Madagascar trade via multiple relationships (Fafchamps and Minten 1999); corporate lawyers are instructed by multiple businesses (Lancaster and Uzzi 2012); suppliers trade with more than one manufacturer (Uzzi 1997); and restaurateurs buy from more than one vendor at fish markets (Kirman and Vriend 2000). Recent theoretical work has shown that with multi-unit demands and supplies, the set of competitive equilibria ${ }^{58}$ has the same lattice structure as with unit demand and supply (Milgrom 2009; Jaume, Massó, and Neme 2012). These results provide a platform

\footnotetext{
${ }^{58}$ With unit demand and unit supply, pairwise stable outcomes, core outcomes, and the competitive equilibria all coinside. This is not true generally. In competitive equilibria a seller must offer the same price to all buyers. While this nondiscrimination constraint never binds in the unit demand and unit supply case, it can bind more generally.
} 
for the approach taken in this paper to be extended to include buyers with heterogeneous demands and suppliers with heterogeneous supplies.

\section{APPENDIX}

\section{A. Size of Inefficiencies}

This section considers the size of inefficiencies, rather than just bounds on the size of inefficiencies, for a simple example: buyer bargaining power $\beta=\frac{1}{2}$ where there is one buyer and two sellers with potential gains from trade $a_{11}$ and $a_{12}$, respectively. All values of $a_{11}$ and $a_{12}$ are considered ( $c$ is normalized to 1). Under investment protocol A, the size of underinvestment inefficiency is shown in Figure A1, panel A for different values of $\gamma$ and $\max \left(a_{11}, a_{12}\right)$. There is no overinvestment inefficiency for $\gamma \in(0,1)$. Under investment protcol B, the size of overinvestment inefficiency is shown in Figure A1, panel B for different values of $a_{11}$ and $a_{12}$.

Underinvestment inefficiency of 100 percent under protocol A is more pervasive than 100 percent overinvestment inefficiency under protocol B. However, when underinvestment inefficiency is not 100 percent it is 0 percent, while for a wide range of parameters there is some overinvestment inefficiency.

\section{B. Proofs}

\section{PROOF OF LEMMA 1:}

It will be assumed that a seller $j^{\prime}$ is removed from the network. The proof for removing a buyer is symmetric. We also assume, for simplicity, that there is a unique match that maximizes the gains from trade following the removal of any agent. This is generically true. The result and proof can be extended to include the non-generic case by comparing the sets of links that might be rematched over following the removal of an agent to the set of possible network decompositions.

Two definitions will be helpful. A chain is a sequence of different links of the following form:

$$
l_{i_{1} j_{2}}, l_{j_{2} i_{2}}, l_{i_{2} j_{3}}, l_{j_{3} i_{3}}, \ldots, l_{i_{k} j_{k}} .
$$

A chain may end at any time as long as preceding links have been in a sequence of this form. A cycle is a sequence of links of the following form:

$$
l_{i_{1} j_{2}}, l_{j_{2} i_{2}}, l_{i_{2} j_{3}}, l_{j_{3} i_{3}}, \ldots, l_{i_{k} j_{k}}, l_{j_{k} i_{1}} .
$$

Now it will be shown that no subset of links from $L_{j^{\prime}}^{+} \cup L_{j^{\prime}}^{-}$can be arranged into a cycle. Suppose, toward a contradiction, that links $\tilde{L}_{j^{\prime}}^{+} \subseteq L_{j^{\prime}}^{+}$and $\tilde{L}_{j^{\prime}}^{\bar{c}} \subseteq L_{j^{\prime}}^{\bar{y}}$ can be arranged into a cycle. Consider the initial (optimal) matching in $L$, before $j^{\prime}$ was removed. As the links in $\tilde{L}_{j^{\prime}}^{+} \cup \tilde{L}_{j^{\prime}}$ can be arranged into a cycle, it is feasible to instead match over the links in $\tilde{L}_{j^{\prime}}^{+}$while no longer matching over the links $\tilde{L}_{j^{\prime}}$, 

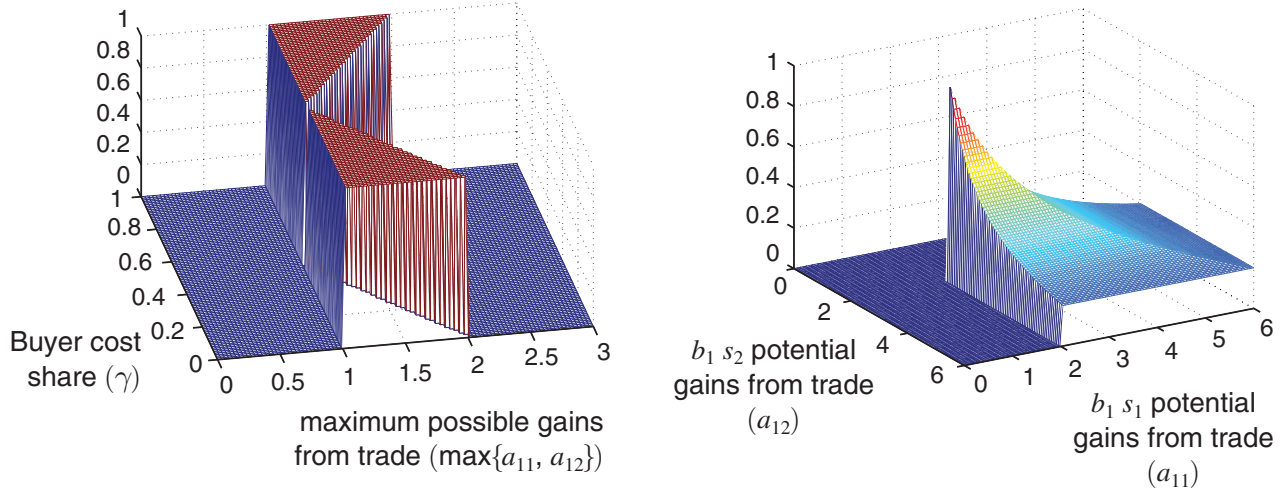

Figure A1. The Size of Inefficiencies with Exogenous and Endogenous Cost Shares

without removing $j^{\prime}$ from the network and without any other rematching. For the initial match to maximize the gains from trade, $\sum_{l \in \tilde{L}_{j^{\prime}}^{+}} \alpha_{l}<\sum_{l \in \tilde{L}_{j^{\prime}}} \alpha_{l^{\prime}}$. Consider now the optimal matching in $L \backslash\left\{j^{\prime}\right\}$. Relative to the initial matching, in this optimal match the links in $L_{j^{\prime}}^{+}$are matched over and the links in $L_{j^{\prime}}^{-}$are no longer matched over. However, as the links in $\tilde{L}_{j^{\prime}}^{+} \cup L_{j^{\prime}}^{\bar{\prime}}$ can be arranged into a cycle, it is feasible to instead match over the links in $L_{j^{\prime}}^{-}$while no longer matching over the links in $\tilde{L}_{j^{\prime}}^{+}$, without affecting any of the other matches. It then follows that $\sum_{l \in \tilde{L}_{j^{\prime}}^{+}} \alpha_{l}>\sum_{l \in \tilde{L}_{j^{\prime}}} \alpha_{l}$. This is a contradiction. We have proved that no subset of links in $L_{j^{\prime}}^{+} \cup L_{j^{\prime}}$ can be arranged into a cycle.

The same logic establishes that a buyer $i^{\prime}$ cannot rematch when $j^{\prime}$ is removed from $L$ unless $i^{\prime \prime}$ s trade partner in $L\left(\mu^{*}\left(i^{\prime} ; \boldsymbol{\alpha}(L)\right)\right)$ is also rematched (or has been removed). Suppose that $\mu^{*}\left(i^{\prime} ; \boldsymbol{\alpha}(L)\right)$ is neither rematched nor removed from the network, but $i^{\prime}$ is rematched. Consider the rematching chain starting from $i^{\prime}$. For the network $L \backslash\left\{j^{\prime}\right\}$, this rematching could be undone and every person in the chain instead matched to their trade partner in $L$, without affecting any of the other rematchings. As it was not optimal to implement the rematching starting at $i^{\prime}$ in the network $L$, it cannot be optimal to implement it in the network $L \backslash\left\{j^{\prime}\right\}$ either. This is now shown more formally. Suppose, toward a contradiction, that a link $l_{i^{\prime} j} \in L_{j^{\prime}}^{+}$, but that for all $i, l_{i \mu^{*}\left(i^{\prime}\right)} \notin L_{j^{\prime}}^{+}$, so that $\mu^{*}\left(i^{\prime}\right)$ is not rematched. Denote by $\hat{L}_{j^{\prime}}^{+} \subseteq L_{j^{\prime}}^{+}$and $\hat{L}_{j^{\prime}}^{\bar{C}} \subseteq$ $L_{j^{\prime}}$ the links in the maximum-length chain that can be constructed starting at link $l_{i^{\prime} j}$ using links $l \in L_{j^{\prime}}^{+} \cup L_{j^{\prime}}$. As $\mu^{*}\left(i^{\prime}\right)$ is not rematched, the buyers in this maximum-length chain need not rematch-all other rematches not involving them would still be possible if they did not rematch. Rematching over these links following the removal of $j^{\prime}$ must maximize the gains from trade, and so $\sum_{l \in \hat{L}_{j^{\prime}}^{+}} \alpha_{l}>\sum_{l \in \hat{L}_{j^{\prime}}} \alpha_{l}$. However, this rematch was possible in the initial network but not implemented, and so: $\sum_{l \in \hat{L}_{j^{\prime}}^{+}} \alpha_{l}<\sum_{l \in \hat{L}_{j^{\prime}}^{-}} \alpha_{l}$. This is a contradiction. 
It follows that the links $l \in L_{j^{\prime}}^{+} \cup L_{j^{\prime}}^{\bar{y}}$ can be arranged into a chain starting with link $l_{j^{\prime} \mu *\left(j^{\prime}\right)}$. Relabeling buyers and sellers, set $j_{1}=j^{\prime}$ and denote this chain by

$$
S\left(j_{1}\right):=\left(l_{j_{1} i_{1}}, l_{i_{1} j_{2}}, l_{j_{2} i_{2}}, l_{i_{2} j_{3}}, \ldots\right) .
$$

It is helpful to introduce some notation for "subchains." Let $S_{j_{k}}\left(j_{1}\right)$ denote the subsequence of $S\left(j_{1}\right)$ containing all links up to but not including $l_{j_{k} i_{k}}$, and let $S^{j_{k}}\left(j_{1}\right)$ denote the subsequence of $S\left(j_{1}\right)$ containing the link $l_{j_{k} i_{k}}$ and all links after it. Thus, $S\left(j_{1}\right)=\left(S_{j_{k}}\left(j_{1}\right), S^{j_{k}}\left(j_{1}\right)\right)$. The remainder of the proof will establish that, if a seller $j_{k}$ is rematched following the removal of $j_{1}$, then $S\left(j_{k}\right)=S^{j_{k}}\left(j_{1}\right)$.

Define the gains from rematching over the sequence $S(j)$ if $j$ is removed from $L$ by

$$
G R(S(j)):=\sum_{l \in L_{j}^{+}} \alpha_{l}-\sum_{l \in L_{j}^{-} / l_{\mu^{*}(j) j}} \alpha_{l} .
$$

It will now be shown that if seller $j_{k}$ rematches in $S\left(j_{1}\right)$, then $j_{1}$ cannot rematch in $S\left(j_{k}\right)$. Suppose, toward a contradiction, that $l_{j_{k} i_{k}} \in S\left(j_{1}\right)$, so that $j_{k}$ is rematched when $j_{1}$ is removed, and that $l_{j_{1} i_{1}} \in S\left(j_{k}\right)$, so that $j_{1}$ is rematched when $j_{k}$ is removed. As $j_{1}$ is rematched when $j_{k}$ is removed, the subsequence of rematchings $S^{j_{1}}\left(j_{k}\right)$ is a possible rematching when $j_{1}$ is removed. However, this rematching must generate weakly lower gains from rematching than the optimal rematch:

$$
G R\left(S^{j_{1}}\left(j_{k}\right)\right) \leq G R\left(S\left(j_{1}\right)\right)=G R\left(S_{j_{k}}\left(j_{1}\right)\right)+G R\left(S^{j_{k}}\left(j_{1}\right)\right) .
$$

Similarly, as $j_{k}$ is rematched when $j_{1}$ is removed, the subsequence of rematchings $S^{j_{k}}\left(j_{1}\right)$ is a possible rematching when $j_{k}$ is removed. However, this rematching must generate weakly lower gains from rematching than the optimal rematch:

$$
G R\left(S^{j_{k}}\left(j_{1}\right)\right) \leq G R\left(S\left(j_{k}\right)\right)=G R\left(S_{j_{1}}\left(j_{k}\right)\right)+G R\left(S^{j_{1}}\left(j_{k}\right)\right)
$$

Combining equations A1 and A2: $G R\left(S_{j_{k}}\left(j_{1}\right)\right)+G R\left(S_{j_{1}}\left(j_{k}\right)\right) \geq 0$. However, the sequence of links $\left(S_{j_{k}}\left(j_{1}\right), S_{j_{1}}\left(j_{k}\right)\right)$ is a cycle. As the gains from rematching over this cycle are weakly positive and this rematching is feasible in the initial network, the initial match cannot have (uniquely) maximized the gains from trade. This is a contradiction.

Thus, $j_{1}$ cannot rematch in $S\left(j_{k}\right)$ if seller $j_{k}$ rematches in $S\left(j_{1}\right)$. An equivalent argument establishes that for all $k^{\prime}<k, j_{k^{\prime}}$ cannot rematch in $S\left(j_{k}\right)$ if seller $k$ rematches in $S\left(j_{1}\right)$.

This result will now be used to show that if $j_{k}$ is rematched following the removal of $j_{1}$, then the same subsequent rematching would be optimal if $j_{k}$ were removed instead of $j_{1}$. In other words, $S\left(j_{k}\right)=S^{j_{k}}\left(j_{1}\right)$. As the rematch following the removal of $j_{k}$ is optimal, $G R\left(S\left(j_{k}\right)\right) \geq G R\left(S^{j_{k}}\left(j_{1}\right)\right)$. However, as there is no $k^{\prime}<k$ such that $j_{k^{\prime}}$ can rematch in $S\left(j_{k}\right)$, the rematch $\left(S_{j_{k}}\left(j_{1}\right), S\left(j_{k}\right)\right)$ following the removal of $j_{1}$ is feasible. By the optimality of the rematch following the removal of $j_{1}$, $G R\left(S_{j_{k}}\left(j_{1}\right)\right)+G R\left(S^{j_{k}}\left(j_{1}\right)\right) \geq G R\left(S_{j_{k}}\left(j_{1}\right)+G R\left(S\left(j_{k}\right)\right)\right.$. Combining the inequalities, $G R\left(S\left(j_{k}\right)\right)=G R\left(S^{j_{k}}\left(j_{1}\right)\right)$ and $S\left(j_{k}\right)=S^{j_{k}}\left(j_{1}\right)$.

Using the symmetry of buyers and sellers and the fact that each agent is rematched (by definition) to his outside trade partner when his trade partner is removed from 
the network, $L_{\mu^{*}(k)}^{+}=L_{k}^{d l}$ and $L_{\mu^{*}(k)}^{-}=L_{k}^{s l} \cup\left\{l_{k \mu^{*}(k)}\right\}$. Furthermore, it has already been shown that the links in $L_{\mu^{*}(k)}^{+} \cup L_{\mu^{*}(k)}^{-}$can be arranged into a chain (and do not cycle). It now follows that outside option chains do not cycle either.

PROOF OF THEOREM 1:

Let $j=\mu^{*}(i)$.

$$
\underline{u}_{i}=\alpha_{i j}-\bar{v}_{j}=\alpha_{i j}-\left(\sum_{l \in L_{j}^{-}} \alpha_{l}-\sum_{l \in L_{j}^{+}} \alpha_{l}\right)=\sum_{l \in L_{i}^{d l}} \alpha_{l}-\sum_{l \in L_{i}^{s l}} \alpha_{l} .
$$

The first equality in equation A3 follows from the lattice structure of core payoffs - buyer $i$ receives his outside option (his minimum core payoff) when $j$ receives his maximum core payoff-as matched buyer-seller pairs split their gains from trade. The second equality follows from Démange (1982) and Leonard (1983), who show that in this environment an agent's maximum core payoff is equal to his marginal contribution to total surplus. The final equality follows from Lemma 1. Equivalent calculations establish the result for sellers' outside options.

\section{PROOF OF COROLLARY 1:}

From the lattice structure of the core, $\bar{v}_{\eta(i)}=\alpha_{\mu^{*}(\eta(i)) \eta(i)}-\underline{u}_{\mu^{*}(\eta(i))}$. From Lemma $1, \mu^{*}(\eta(i))$ 's outside option chain will be a proper subset of $i$ 's outside option chain. Theorem 1 can then be used to relate $i$ 's and $\mu^{*}(\eta(i))$ 's outside option payoffs to each other: $\underline{u}_{i}=\alpha_{i \eta(i)}-\alpha_{\mu^{*}(\eta(i)) \eta(i)}+\underline{u}_{\mu^{*}(\eta(i))}$. Combining the above equalities, $\bar{v}_{\eta(i)}+\underline{u}_{i}=\alpha_{i \eta(i)}$. As $\bar{v}_{\eta(i)} \geq v_{\eta(i)}$ whenever $\eta(i)$ receives a core payoff, $v_{\eta(i)}+\underline{u}_{i} \leq \alpha_{i \eta(i)}$. Thus, if $i$ ever received a payoff less than $\underline{u}_{i}, i$, and $\eta(i)$ would have a profitable deviation by trading with each other. An equivalent calculation can be shown for a seller $j$.

\section{PROOF OF PROPOSITION 1:}

Proposition 1 will be proved through two lemmas. First, it will be shown that efficiency losses due to overinvestment are bounded.

LEMMA 2: For exogenous cost shares $\gamma \in(0,1)$ and homogeneous potential gains from trade a where $a_{i j} \in\{0,1\}$, there is no overinvestment inefficiency. For any (heterogeneous) potential gains from trade a, overinvestment inefficiency is bounded by the amounts shown in Table A1, and this bound is tight.

This proof is undertaken in five parts. In Part (i) the homogeneous gains from the trade case is considered. Part (ii) corresponds to the first row in Table A1, part (iii) to the second row, part (iv) to rows five and six, and part (v) to rows three, four, seven, and eight. In each part the existence of the bound and its tightness is proved.

Before beginning, note that when $\beta \in(0,1)$ and $\gamma \in(0,1)$, all non-trade links must benefit both parties and, as outside option chains cannot cycle (Lemma 1), each component with any non-trade links must take the form of a chain, as shown in Figure A2 for the case of four trade links. This insight underlies the proofs of parts (i) and (ii). 
TABLE A1-BOUNDING OVERINVESTMENT INEFFICIENCY

\begin{tabular}{lcc}
\hline \hline$\beta$ & $\gamma$ & Bound on overinvestment inefficiency \\
\hline$\in(0,1)$ & $\in(0,1)$ & $h(\tilde{K}, \gamma, \beta) \leq \frac{1}{2}$ \\
$\in\{0,1\}$ & $\in(0,1)$ & 0 \\
0 & 0 & 0 \\
$\in(0,0.5)$ & 0 & $\frac{\beta}{1-\beta}$ \\
$\in[0.5,1]$ & 0 & 1 \\
$\in[0,0.5]$ & 1 & 1 \\
$\in(0.5,1)$ & 1 & $\frac{1-\beta}{\beta}$ \\
1 & 1 & 0 \\
\hline
\end{tabular}

Notes: Where $\tilde{K}=\min \{m, n\}$ the number of agents on the short side of the market and $h(\tilde{K}, \gamma, \beta)$

$:=(\tilde{K}-1)\left(\max \left\{\frac{(\tilde{K}-1)+(1-\gamma)}{1-\beta}+\frac{(\tilde{K}-1)(1-\gamma)}{\beta}, \frac{(\tilde{K}-1)+(\gamma)}{\beta}+\frac{(\tilde{K}-1)(\gamma)}{1-\beta}\right\}-\tilde{K}\right)^{-1}$.

When $\beta \in\{0,1\}$ and $\gamma \in(0,1)$ the unique stable network is the empty network.

Part (i): Consider a component with non-trade links. With homogeneous gains from trade, Theorem 1 can be directly applied to show that $\underline{u}_{i}=0$ for all buyers in the component and $\underline{v}_{j}=0$ for all sellers in the component. Thus, there are insufficient incentives for any outside option links to be formed, and there is no outside overinvestment inefficiency in any stable network.

Part (ii): Label buyers and sellers as in Figure A1, but with $\tilde{K}$ buyers and $\tilde{K}$ sellers. As neither $b_{\tilde{K}}$ nor $s_{1}$ has an outside trade partner $\underline{u}_{\tilde{K}}=0$ and $\underline{v}_{1}=0$, and so $u_{\tilde{K}}=\beta \bar{u}_{\tilde{K}}$ and $v_{1}=(1-\beta) \underline{v}_{1}$. For buyer $b_{\tilde{K}}$ and seller $s_{1}$ to then have sufficient incentives to form their trade links we must have

$$
\bar{v}_{1} \geq \frac{(1-\gamma) c}{1-\beta}, \quad \bar{u}_{\tilde{K}} \geq \frac{\gamma c}{\beta} .
$$

Applying Theorem 1,

$$
\begin{aligned}
& \bar{v}_{1}=\sum_{k=2}^{j}\left(\alpha_{k-1, k-1}-\alpha_{k-1, k}\right)+\bar{v}_{j} \geq \frac{(1-\gamma) c}{(1-\beta)}, \\
& \bar{u}_{\tilde{K}}=\sum_{k=i+1}^{\tilde{K}}\left(\alpha_{k, k}-\alpha_{k-1, k}\right)+\bar{u}_{i} \geq \frac{\gamma c}{\beta},
\end{aligned}
$$

for $j \in\{2, \ldots, \tilde{K}\}$ and $i \in\{1, \ldots, \tilde{K}-1\}$.

All buyers other than $b_{\tilde{K}}$ and all sellers other than $s_{1}$ must have sufficient incentives to form their outside option links. Thus, for $j \neq 1$, $\beta \underline{v}_{j}+(1-\beta) \bar{v}_{j}-(1-\beta) \bar{v}_{j} \geq(1-\gamma) c ; \quad$ and similarly for $i \neq \tilde{K}$, $(1-\beta) \underline{u}_{i} \geq \gamma c$. Applying Theorem 1 again,

$$
\begin{aligned}
& \underline{u}_{i}=\sum_{k=i+1}^{\tilde{K}}\left(\alpha_{k-1, k}-\alpha_{k, k}\right) \geq \frac{\gamma c}{(1-\beta)}, \\
& \underline{v}_{j}=\sum_{k=2}^{j}\left(\alpha_{k-1, k}-\alpha_{k-1, k-1}\right) \geq \frac{(1-\gamma) c}{\beta},
\end{aligned}
$$


for $j \in\{2, \ldots, \tilde{K}\}$ and $i \in\{1, \ldots, \tilde{K}-1\}$. Combining equations (A5), (A6), (A7), and $(\mathrm{A} 8)$,

$$
\begin{aligned}
& \bar{v}_{j} \geq \frac{(1-\gamma) c}{(1-\beta)}+\sum_{k=2}^{j}\left(\alpha_{k-1, k}-\alpha_{k-1, k-1}\right) \geq \frac{(1-\gamma) c}{(1-\beta)}+\frac{(1-\gamma) c}{\beta}, \\
& \bar{u}_{i} \geq \frac{\gamma c}{\beta}+\sum_{k=i+1}^{\tilde{K}}\left(\alpha_{k-1, k}-\alpha_{k, k}\right) \geq \frac{\gamma c}{\beta}+\frac{\gamma c}{(1-\beta)},
\end{aligned}
$$

for $j \in\{2, \ldots, \tilde{K}\}$ and $i \in\{1, \ldots, \tilde{K}-1\}$. The gains from trade in a network are equal to the total payoffs received by all parties. Combining equations (A4), (A7), and (A10) and then equations (A4), (A8), and (A10):

$$
\begin{aligned}
\sum_{k=1}^{\tilde{K}} \alpha_{k, k} & =\sum_{k=1}^{\tilde{K}}\left(\underline{u}_{k}+\bar{v}_{k}\right) \geq(\tilde{K}-1)\left(\frac{1}{(1-\beta)}+\frac{1-\gamma}{\beta}\right) c+\frac{1-\gamma}{1-\beta} c \\
& :=f_{1}(\tilde{K}, \gamma, \beta) c \\
\sum_{k=1}^{\tilde{K}} \alpha_{k, k} & =\sum_{k=1}^{\tilde{K}}\left(\bar{u}_{k}+\underline{v}_{k}\right) \geq(\tilde{K}-1)\left(\frac{1}{\beta}+\frac{\gamma}{(1-\beta)}\right) c+\frac{\gamma}{\beta} c:=f_{2}(\tilde{K}, \gamma, \beta) c .
\end{aligned}
$$

This provides a lower bound on the gains from trade reached with $\tilde{K}$ trade links. The net gains from trade in the network if all outside options links were removed would be at least $\max \left\{f_{1}(\tilde{K}, \gamma, \beta) c, f_{2}(\tilde{K}, \gamma, \beta) c\right\}-\tilde{K} c$. As $(\tilde{K}-1) c$ is invested in outside option links, this provides following upper bound on overinvestment inefficiency

$$
h(\tilde{K}, \gamma, \beta):=(\tilde{K}-1)\left(\max \left\{f_{1}(\tilde{K}, \gamma, \beta), f_{2}(\tilde{K}, \gamma, \beta)\right\}-\tilde{K}\right)^{-1} .
$$

Thus, for all stable components with $\tilde{K}$ matches, all levels of buyer bargaining power $\beta \in(0,1)$, and all buyer cost shares $\gamma \in(0,1), O I I \leq h(\tilde{K}, \gamma, \beta)$.

To show that OII is always less than 50 percent, the bound derived above can be maximized over $\beta$ and $\gamma: \overline{O I I}:=\max _{\beta, \gamma}\{h(\tilde{K}, \gamma, \beta)\}=h\left(\tilde{K}, \frac{1}{2}, \frac{1}{2}\right)=\frac{1}{2}$. This is independent of $\tilde{K}$.

It will now be shown that the bound derived above is tight. Consider a component with $\tilde{K}$ matches and $\beta \geq \gamma$, which implies that $f_{1} \geq f_{2}$. Set $\alpha_{11}=c /(1-\beta), \alpha_{k, k}=c /(1-\beta)+(1-\gamma) c / \beta$ for all $k \in\{2, \tilde{K}\}$, and $\alpha_{k-1, k}=c /(1-\beta)+(1-\gamma) c / \beta$ for all $k \in\{2, \ldots, \tilde{K}\}$, and set the value of all other links to 0 . It can be verified that for these potential gains from trade, that the chain network of Figure A2 is stable and overinvestment inefficiency achieves the upper bound. Similar examples can be constructed when $\beta \leq \gamma$, which implies that $f_{2} \geq f_{1}$.

So far, an $O I I$ bound has been found for formed components with $\tilde{K}$ matches, and it has been shown that this bound is tight. To find the tight $O I I$ bound for any stable network that $m$ buyers and $n$ sellers might form, the following possibilities need to be considered: (i) multiple components being formed; or (ii) a single component being formed, but with only a proper subset of the short side of the market matched. 


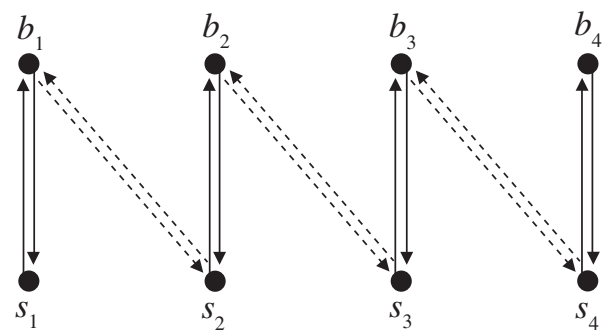

Figure A2. Component Structure: Four Trade Links

However, it will now be shown that for any stable network $m$ buyers and $n$ sellers could form, the maximum possible level of $O I I$ occurs when there is a single component with $\tilde{K}=\min \{m, n\}$ matches - the maximum possible number of matches. This is true for any $\beta \in(0,1)$ and any $\gamma \in(0,1)$.

If $\beta>(\geq) \gamma$, then $f_{1}>(\geq) f_{2}$ and

$$
\frac{\partial \frac{\tilde{K}-1}{f_{1}(\tilde{K}, \gamma, \beta)-\tilde{K}}}{\partial \tilde{K}}>(\geq) 0 .
$$

If $\beta<(\leq) \gamma$, then $f_{2}>(\geq) f_{1}$ and

$$
\frac{\partial \frac{\tilde{K}-1}{f_{2}(\tilde{K}, \gamma, \beta)-\tilde{K}}}{\partial \tilde{K}}>(\geq) 0 .
$$

Thus, $h(\tilde{K}, \gamma, \beta)$ is weakly increasing in $\tilde{K}$, and the $O I I$ bound for a network with $m$ buyers and $n$ sellers is highest when there are as many matches as possible: $\tilde{K}=\min \{m, n\}$.

Part (iii): As $\gamma \in(0,1)$, the component structure of any nonempty network with overinvestment inefficiency must be of the form shown in Figure A2. In any such component there exists a seller and a buyer both of whom have no outside trade partner. If $\beta=1$, then the seller will have a profitable deviation to delete his trade link as he contributes toward the cost of its formation but receives none of the gains from trade. If $\beta=0$, then the buyer will not be willing to form his trade link. The unique stable network is the empty network, and there is never any overinvestment.

Part (iv): When $\left(\beta \geq \frac{1}{2}\right.$ and $\left.\gamma=0\right)$ or $\left(\beta \leq \frac{1}{2}\right.$ and $\left.\gamma=1\right)$, there exist potential gains from trade for which the efficiency loss in all stable networks is 100 percent. This is shown for $\beta \leq 1 / 2$ and $\gamma=1$ in Figure A3.

The efficiency loss is $\frac{c}{2 c+2 \varepsilon-c} \rightarrow 1$ as $\varepsilon \rightarrow 0$. A symmetric example can be constructed for $\beta \geq \frac{1}{2}$ and $\gamma=0$, with the roles of buyers and sellers reversed.

Part (v): Let $\beta<\frac{1}{2}$ and $\gamma=0$. As $\gamma=0$, only sellers can form outside option links. By Theorem 1 , seller $s_{j}$ 's incentives to form an outside option link to buyer $b_{i}$ are given by $\beta \alpha_{\eta(j) j}-\bar{u}_{\eta(j)} \leq \beta \alpha_{\eta(j) j}$. Thus, the maximum 
Panel A. Potential links

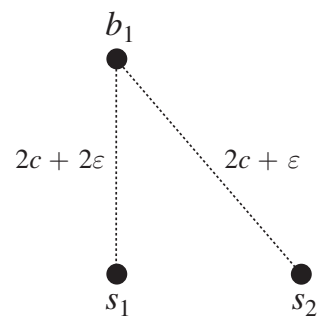

Panel B. Efficient network

$u_{1}=\beta(2 c+2 \varepsilon)-c$

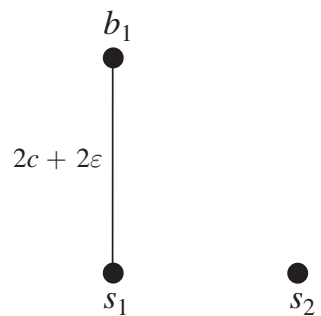

$v_{1}=(1-\beta)(2 c+2 \varepsilon)$
Panel C. Stable network

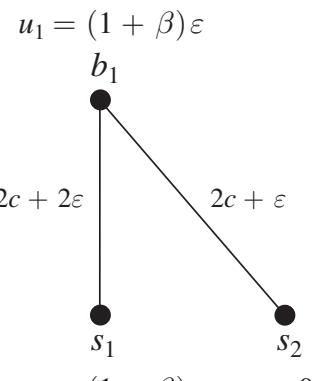

Figure A3. OverinVestment InefFiciency of 100 PerCent

incentives to form outside option links can always occur in networks where sellers' outside trade partners do not trade with anyone and $\beta\left(\alpha_{\eta(j) j}-\bar{u}_{\eta(j)}\right)$ $=\beta \alpha_{\eta(j) j}$. Without loss of generality, therefore, and as sellers will form at most one outside option link, inefficiency in network components consisting of two buyers and one seller can be considered: If an overinvestment inefficiency bound for all stable networks is found in these networks, it will apply to all networks.

Consider then, without loss of generality, ${ }^{59}$ the network component shown in Figure A4 In this network component, in order for there to be any efficiency loss due to overinvestment in non-trade links, $s_{1}$ must form links to both $b_{1}$ and $b_{2}$. To be incentivized to do this, $s_{1}$ 's payoff in the network shown in Figure A3, panel C must be greater than his payoff in the efficient network: $\zeta+(1-\beta) \varepsilon-2 c \geq(1-\beta)$ $\times(\zeta+\varepsilon)-c$. This holds if and only if $\zeta \beta \geq c$. Let $c=\zeta \beta-\xi, \xi \geq 0$, so that this constraint is satisfied. For this cost, and for the gains from trade above, $O I I=\frac{\zeta \beta-\xi}{\zeta(1-\beta)+\varepsilon+\xi}$. The efficiency loss in the worst stable network is maximized by setting $\xi=\varepsilon=0$. Thus, overinvestment inefficiency must be less than or equal to $\frac{\beta}{1-\beta}$ for any network with $\beta<\frac{1}{2}$ and $\gamma=0$. This bound is achieved in the above example with $\xi=\varepsilon=0$.

It will now be shown that losses due to underinvestment inefficiency cannot be bounded below 100 percent.

LEMMA 3: For exogenous cost shares, all nonempty sets $P, Q$ of buyers and sellers, respectively, and for all $\beta \neq \gamma$, there exist potential gains from trade a for which all the net gains from trade generated by the efficient network are lost to underinvestment inefficiency in all stable networks. When $\beta=\gamma$, in contrast, there is no underinvestment inefficiency in any stable network for any potential gains from trade $\mathbf{a}$.

Suppose $\beta=\gamma \cdot{ }^{60}$ For any potential gains from trade a, suppose, toward a contradiction, that a link $l_{i j}$ is inefficiently underinvested in, in a stable network $L$. From

\footnotetext{
${ }^{59}$ Varying $\varepsilon \geq 0$ and $\zeta>0$, this example accounts for all possible two-buyers one-seller networks, for any $c$.

${ }^{60}$ A similar condition is found in Caballero and Hammour (1998).
} 

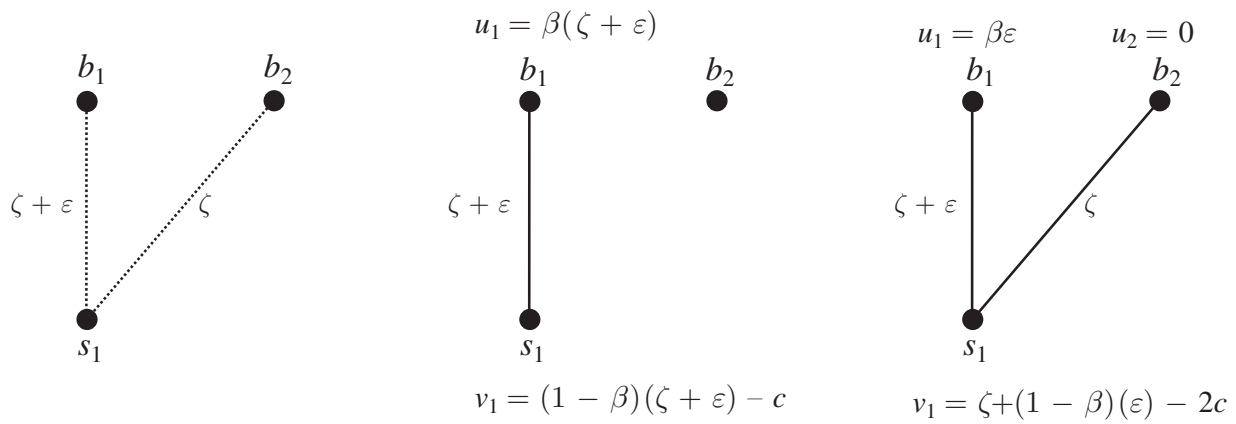

Figure A4. Bounded Overinvestment INEFFICIENCY

the definition of underinvestment inefficiency, $a_{i j}>c$. However, this implies that $i$ would benefit from forming this link as $\beta a_{i j}>\gamma c$, and $j$ would also benefit from forming this link as $(1-\beta) a_{i j}>(1-\gamma) c$. Network $L$ is then not stable, which is a contradiction.

Suppose $\beta \neq \gamma$. Consider any gains from trade a with an associated nonempty efficient network $L^{e}(\mathbf{a})$. Adjust the value of the links in a as follows (and denote these adjusted gains from trade $\hat{\mathbf{a}})$. For $\boldsymbol{\alpha}=\mathbf{a}$, find the matching $\mu^{*}(\boldsymbol{\alpha})$ that maximizes the gains from trade. Set $\hat{a}_{i \mu^{*}(i)} \in(c, \max \{\gamma c / \beta,(1-\gamma) c /(1-\beta)\})$ for all $i \in P$. Finally, set $\hat{a}_{i j}<c$ for all other links (for all $j \neq \mu^{*}(i)$ ). As $\beta \neq \gamma$, $\max \{\gamma c / \beta,(1-\gamma) c /(1-\beta)\}>c$. The efficient network is therefore the nonempty network $L^{e}(\mathbf{a})=L^{e}(\mathbf{a})$.

Suppose link $l_{i \mu^{*}(i)}$ is formed. As it will never be profitable for a buyer and seller to form a link that generates less surplus than it costs, this will be $i$ 's only link and $\mu^{*}(i)$ 's only link. From Theorem 1 and equation 5, buyer $i$ 's payoff will be $\beta \hat{a}_{i \mu^{*}(i)}-\gamma c$, while seller $\mu^{*}(i)$ 's payoff will be $(1-\beta) \hat{a}_{i \mu^{*}(i)}-(1-\gamma) c$. It is easily verified that if $a_{i \mu^{*}(i)}<\max \{\gamma c / \beta,(1-\gamma) c /(1-\beta)\}$, either $i$ or $\mu^{*}(i)$ will receive a negative payoff and have a profitable deviation to not invest in $l_{i \mu^{*}(i)}$. The link $l_{i \mu^{*}(i)}$ can then never be formed in a stable network, and the unique stable network is the empty network.

We now show that the empty network satisfies stronger stability requirements than pairwise Nash stability. A strong Nash equilibrium of a game $G$ played by players $N$ is a strategy profile $\sigma$ such that there is no coalition $M \subseteq N$ and no other strategy profile $\sigma^{\prime}$ in which

- $\sigma_{i}^{\prime}=\sigma_{i}$ for all $i \notin M$;

- each $i \in M$ strictly prefers $\sigma^{\prime}$ to $\sigma$. 
Note that in a strong Nash equilibrium a buyer $i$ still chooses which links to invest in (his strategy set is still $\{0,1\}^{n}$ ), and his payoff from a formed network is still given by equation 5 less his costs of link formation.

COROLLARY 5: For gains from trade $\hat{\mathbf{a}}$ defined in the Proof of Lemma 3 and $\gamma \neq \beta$, the unique strong Nash equilibrium of the simultaneous link formation game is the empty network.

Any network in which a link $l_{i j} \neq l_{i \mu^{*}(i)}$ is formed cannot be a strong Nash equilibrium. As already shown either $i$ or $j$ will have an individual profitable deviation deleting it. Given this, a network in which link $l_{i \mu^{*}(i)}$ is formed cannot be a strong Nash equilibrium either. As argued above, either $i$ or $\mu^{*}(i)$ will have an individual profitable deviation deleting it. However, the empty network is a strong Nash equilibrium. Although there are net gains from trade that can be realized by forming links $i$ and $\mu^{*}(i)$, all such deviations leave either $i$ or $\mu^{*}(i)$ with a negative payoff. As agents choose only the set of links to invest in (and not how much to invest), there is no way that the agent who receives the negative payoff can be compensated for his negative payoff.

\section{PROOF OF PROPOSITION 2:}

Part (i): Suppose that there is underinvestment in link $l_{i j}$, so that $a_{i j}>c$. Buyer $i$ and seller $j$ will then have a profitable pairwise deviation to form link $l_{i j}$ and pay investment shares $\gamma_{i j}=\beta$.

Part (ii): This proof is by counterexample. Consider gains from trade a such that if the complete network is formed ( $\alpha=\mathbf{a})$, buyers $i \in P^{\prime} \subseteq P$ will be matched and for each $i \in P^{\prime}, a_{i \mu^{*}(i)}=1, a_{i \eta(i)}=1-\varepsilon$, and $a_{k \eta(i)}=a_{k \mu^{*}(i)}=0$ for all $k \neq i$. Set the value of all other gains from trade to zero. Let $c=\frac{1}{2}-\varepsilon$ and consider first $\beta \in\left[0, \frac{1}{2}\right]$. The efficient network, consisting of the links $\left\{l_{i \mu(i)}\right\}_{i \in P^{\prime}}$, will not be stable for any such $\beta$ : $i$ will increase his share of the surplus from trade with $\mu^{*}(i)$ by $(1-\beta) a_{i \mu^{*}(i)}=(1-\beta)(1-\varepsilon)$, at a cost of $c=\frac{1}{2}-\varepsilon$, if he forms the link to $\eta(i)$. This increases $i$ 's net payoff if and only if $(1-\beta)(1-\varepsilon)>\frac{1}{2}-\varepsilon$ (equivalently $\beta<1 /(2-2 \varepsilon) \leq 1 / 2)$. It is then straightforward to show that in the unique stable network, $i$ pays the entire cost of forming the link $l_{i \eta(i)}$ and pays between $1 / 2-(2-\beta) \varepsilon$ and $1 / 2-\varepsilon$ toward the formation of link $l_{i \mu(i)}$. The efficiency loss in the unique stable network is therefore $(0.5-\varepsilon) /(0.5+\varepsilon)$, and as $\varepsilon \rightarrow 0$ the efficiency loss goes to 100 percent. To show that the efficiency loss can go to 100 percent in all stable networks with $\beta \in\left[\frac{1}{2}, 1\right]$, relabel $i \in P$ as $j \in Q$, $\mu^{*}(i)$ as $\mu^{*}(j)$, and $\eta(i)$ as $\eta(j)$.

\section{PROOF OF COROLLARY 3:}

If

$$
\max _{i, j} \alpha_{i j}<\max \left\{\frac{\gamma}{\beta} c, \frac{1-\gamma}{1-\beta} c\right\}
$$


then for all $i, j$ pairs, either $\beta \alpha_{i j}<\gamma c$ and $i$ does not want to form a link to $j$ or $(1-\beta) \alpha_{i j}<(1-\gamma) c$ and $j$ does not want to form a link to $i$. The empty network will be stable, and there will be 100 percent underinvestment inefficiency. If instead there exist $i$ and $j$ such that $\beta \alpha_{i j} \geq \gamma c$ and $(1-\beta) \alpha_{i j} \geq(1-\gamma) c$, then the empty network cannot be stable: $i$ and $j$ will have a profitable deviation by forming a link to each other. Together, these conditions imply that there cannot be 100 percent underinvestment inefficiency if

$$
\max _{i, j} \alpha_{i j} \geq \max \left\{\frac{\gamma}{\beta} c, \frac{1-\gamma}{1-\beta} c\right\} .
$$

\section{PROOF OF COROLLARY 4:}

As before, let $\tilde{K}=\min \{m, n\}$,

$$
\begin{gathered}
f_{1}(\gamma, \beta, \tilde{K})=\frac{(\tilde{K}-1)+1-\gamma}{1-\beta}+\frac{(\tilde{K}-1)(1-\gamma)}{\beta}, \\
f_{2}(\gamma, \beta, \tilde{K})=\frac{(\tilde{K}-1)+\gamma}{\beta}+\frac{(\tilde{K}-1) \gamma}{1-\beta} .
\end{gathered}
$$

By definition, $h(\gamma, \beta, m, n)=(\tilde{K}-1) /\left(\max \left\{f_{1}(\gamma, \beta, \tilde{K}), f_{2}(\gamma, \beta, \tilde{K})\right\}-\tilde{K}\right)$. Note that $f_{1}(\gamma, \beta, \tilde{K})$ is decreasing in $\gamma$, while $f_{2}(\gamma, \beta, \tilde{K})$ is increasing in $\gamma$, so it is when $f_{1}(\gamma, \beta, \tilde{K})=f_{2}(\gamma, \beta, \tilde{K})$ that $\gamma$ minimizes $\max \left\{f_{1}(\gamma, \beta, \tilde{K}), f_{2}(\gamma, \beta, \tilde{K})\right\}$ (and hence maximizes $h(\gamma, \beta, m, n))$. Observing that $f_{1}(\gamma, \beta, \tilde{K})=f_{2}(\gamma, \beta, \tilde{K})$ when $\gamma=\beta$ completes the proof.

\section{REFERENCES}

Abreu, Dilip, and Mihai Manea. 2012a. "Bargaining and efficiency in networks." Journal of Economic Theory 147 (1): 43-70.

-Abreu, Dilip, and Mihai Manea. 2012b. "Markov equilibria in a model of bargaining in networks." Games and Economic Behavior 75 (1): 1-16.

Acemoglu, Daron, and Robert Shimer. 1999. "Holdups and efficiency with search frictions." International Economic Review 40 (4): 827-49.

Andrade, Gregor, Mark Mitchell, and Erik Stafford. 2001. "New Evidence and Perspectives on Mergers." Journal of Economic Perspectives 15 (2): 103-20.

Aumann, Robert J. 1959. "Acceptable Points in General Cooperative n-Person Games." Contributions to the Theory of Games, Vol. 4. edited by A. W. Tucker and R. D. Luce, 287-324. Princeton: Princeton University.

Bloch, Francis, and Matthew Jackson. 2006. "Definitions of equilibrium in network formation games." International Journal of Game Theory 34 (3): 305-18.

Bloch, Francis, and Matthew O. Jackson. 2007. "The formation of networks with transfers among players." Journal of Economic Theory 133 (1): 83-110.

Blume, Larry, David Easley, Jon Kleinberg, and Eva Tardos. 2009. "Trading networks with price-setting agents." Games and Economic Behavior 67 (1): 36-50.

Brandenburger, Adam M., and Barry J. Nalebuff. 1996. Co-opetition. New York: Doubleday.

Caballero, Ricardo J., and Mohamad L. Hammour. 1998. “The Macroeconomics of Specificity.” Journal of Political Economy 106 (4): 724-67.

Caplin, Andrew, and John V. Leahy. 2010. "Comparative Statics in Markets for Indivisible Goods." National Bureau of Economic Research (NBER) Working Paper 16285. 
Caplin, Andrew, and John V. Leahy. 2014. "A Graph Theoretic Approach to Markets for Indivisible Goods." Journal of Mathematical Economics 52: 112-22.

- Charness, Gary, Margarida Corominas-Bosch, and Guillaume Fréchette. 2007. "Bargaining and network structure: An experiment." Journal of Economic Theory 136 (1): 28-65.

- Cole, Harold L., George J. Mailath, and Andrew Postlewaite. 2001a. "Efficient Non-Contractible Investments in Finite Economies." Advances in Theoretical Economics 1 (1): 333-73.

-Cole, Harold L., George J. Mailath, and Andrew Postlewaite. 2001b. "Efficient Non-Contractible Investments in Large Economies." Journal of Economic Theory 101 (2): 333-73.

-Corominas-Bosch, Margarida. 2004. "Bargaining in a network of buyers and sellers." Journal of Economic Theory 115 (1): 35-77.

-Currarini, Sergio, and Massimo Morelli. 2000. "Network formation with sequential demands." Review of Economic Design 5 (3): 229-49.

Cutler, Robert M. 2010. "Turkmenistan-China Gas Pipeline Becomes a Reality.” Central Asia-Caucasus Analyst. February.

Dealbook. 2010. "Severstal Receives \$177 Million Breakup Fee from Arcelor." New York Times. July 21. http://dealbook.nytimes.com/2006/07/21/severstal-receives-177-million-breakup-fee-fromarcelor/?_r=0.

Demange, Gabrielle. 1982. "Strategyproofness in the Assignment Market Game." Labratorie d'Econométrie de l'Ecole Polytechnique. Preprint: Paris.

-Demange, Gabrielle, David Gale, and Marilda Sotomayor. 1986. "Multi-item auctions." Journal of Political Economy 94 (4): 863-72.

Elliott, Matthew. 2014. "Heterogeneities and the Fragility of the Labor Market." Unpublished.

Elliott, Matthew, and Francesco Nava. 2014. "Decentralized Bargaining: Efficiency and the Core." http://www.its.caltech.edu/ melliott/papers/decentralized_bargaining.pdf.

-Fafchamps, Marcel, and Bart Minten. 1999. "Relationships and Traders in Madagascar." Journal of Development Studies 35 (6): 1-35.

-Fainmesser, Itay P. 2012. "Community Structure and Market Outcomes: A Repeated Games-inNetworks Approach.” American Economic Journal: Miocroeconomics 4 (1): 32-69.

Fainmesser, Itay, and David A. Goldberg. 2011. "Bilateral and Community Enforcement in a Networked Market with Simple Strategies." http://www.brown.edu/academics/economics/sites/brown. edu.academics.economics/files/uploads/2011-2_paper.pdf.

Felli, Leonardo, and Kevin Roberts. 2002. "Does competition solve the hold-up problem?" Center for Economic Policy Research (CEPR) Discussion Paper 3535.

-Gale, Douglas M., and Shachar Kariv. 2007. "Financial Networks." American Economic Review 97 (2): 99-103.

Gautier, Pieter, and Christian L. Holzner. 2013. "Simultaneous Search and Network Efficiency." http:// feb.kuleuven.be/eng/ew/seminars/papers2013/Paper_Gautier.pdf.

Hall, Robert E., and Alan B. Krueger. 2008. "Wage Formation between Newly Hired Workers and Employers: Survey Evidence.” Institute for the Study of Labor (IZA) Discussion Paper 3775.

- Horn, Henrik, and Asher Wolinsky. 1988. "Bilateral monopolies and incentives for merger." RAND Journal of Economics 19 (3): 408-19.

Howson, Peter. 2003. Due Diligence: The Critical Stage in Mergers and Acquisitions. Burlington, VT: Gower Publishing.

- Iozzi, Alberto, and Tommaso Valletti. 2014. "Vertical Bargaining and Countervailing Power." American Economic Journal: Microeconomics 6 (3): 106-35.

Jackson, Matthew O. 2008. Social and Economic Networks. Princeton: Princeton University Press.

-Jackson, Matthew O., and Asher Wolinsky. 1996. "A Strategic Model of Social and Economic Networks." Journal of Economic Theory 71 (1): 44-74.

- James, Christopher M., and Peggy Wier. 1987. "Returns to Acquirers and Competition in the Acquisition Market: The Case of Banking." Journal of Political Economy 95 (2): 355-70.

- Jarrell, Gregg A., and Annette B. Poulsen. 1989. "The returns to acquiring firms in tender offers: Evidence from three decades." Financial Management 18 (3): 12-19.

- Jaume, Daniel, Jordi Massó, and Alejandro Neme. 2012. "The multiple-partners assignment game with heterogeneous sales and multi-unit demands: Competitive equilibria." Mathematical Methods of Operations Research 76 (2): 161-87.

Kirman, Alan P., and Nicolaas J. Vriend. 2000. "Learning to be Loyal. A Study of the Marseille Fish Market.” In Interaction and Market Structure 484 (2000): 33-56.

Koutsoupias, Elias, and Christos Papadimitriou. 2009. "Worst-case equilibria." Computer Science Review 3 (2): 65-69. 
Kranton, Rachel E., and Deborah F. Minehart. 2000a. "Competition for goods in buyer-seller networks." Review of Economic Design 5 (3): 301-31.

-Kranton, Rachel E., and Deborah F. Minehart. 2000b. "Networks versus vertical integration." RAND Journal of Economics 31 (3): 570-601.

-Kranton, Rachel E., and Deborah F. Minehart. 2001. "A Theory of Buyer-Seller Networks.” American Economic Review 91 (3): 485-508.

Lancaster, Ryon, and Brian Uzzi. 2012. "Legally charged: Embeddedness and profit in large law firm legal billings." Sociological Focus 45 (1): 1-22.

Lee, Robin S., and Kyna Fong. 2013. "Markov-Perfect Network Formation An Applied Framework for Bilateral Oligopoly and Bargaining in Buyer-Seller Networks." http://www.people.fas.harvard. edu/ robinlee/papers/MPNENetworkFormation.pdf.

Leonard, Herman B. 1983. "Elicitation of Honest Preferences for the Assignment of Individuals to Positions." Journal of Political Economy 91 (3): 461-79.

-Manea, Mihai. 2011. "Bargaining in Stationary Networks." American Economic Review 101 (5): 2042-80.

McAfee, R. Preston, and Marius Schwartz. 1994. "Opportunism in Multilateral Vertical Contracting: Nondiscrimination, Exclusivity, and Uniformity." American Economic Review 84 (1): 210-30.

-Milgrom, Paul. 2009. "Assignment Messages and Exchanges." American Economic Journal: Microeconomics 1 (2): 95-113.

-Mitchell, Mark L., and J. Harold Mulherin. 1996. "The impact of industry shocks on takeover and restructuring activity." Journal of Financial Economics 41 (2): 193-229.

-Myerson, R. B. 1977. "Values of Games in Partition Function Form." International Journal of Game Theory 6 (1): 23-31.

-Nava, Francesco. 2015. "Efficiency in Decentralized Oligopolistic Markets." Journal of Economics Theory 157: 315-48.

Nishiguchi, Toshihiro. 1994. Strategic Industrial Sourcing: The Japanese Advantage. Oxford: Oxford University Press.

-Officer, Micah S. 2003. "Termination fees in mergers and acquisitions." Journal of Financial Economics 69 (3): 431-67.

Oravetz, Justin W. 2004. "Is a Merger Agreement Ever Certain: The Impact of the Omnicare Decision on Deal Protection Devices." Delaware Journal of Corporate Law 29 (3).

- Polanski, Arnold. 2007. "Bilateral bargaining in networks." Journal of Economic Theory 134 (1): 557-65.

Quint, T. 1991. "Characterization of Cores of Assignment Games." International Journal of Game Theory 19 (4): 413-20.

Reuters. 2008. "Nabucco pipeline cost rises to 7.9 bln euros." May 29.

Roth, Alvin E., and Marilda A. Oliveira Sotomayor. 1990. Two-Sided Matching: A Study in Game-Theoretic Modeling and Analysis. Cambridge: Cambridge University Press.

- Roughgarden, Tim, and Éva Tardos. 2004. "Bounding the inefficiency of equilibria in nonatomic congestion games." Games and Economic Behavior 47 (2): 389-403.

-Segal, Ilya. 1999. "Contracting with Externalities." Quarterly Journal of Economics 114 (2): 337-88.

-Segal, Ilya R., and Michael D. Whinston. 2000. "Exclusive contracts and protection of investments." RAND Journal of Economics 31 (4): 603-33.

-Shapley, L. S., and M. Shubik. 1972. "The Assignment Game I: The Core." International Journal of Game Theory 1: 111-30.

-Shleifer, Andrei, and Robert W. Vishny. 1986. "Greenmail, white knights, and shareholders' interest." RAND Journal of Economics 17 (3): 293-309.

Uzzi, Brian. 1997. "Social Structure and Competition in Interfirm Networks: The Paradox of Embeddedness." Administrative Science Quarterly 42 (1): 35-67.

-Wälde, Thomas W. 2008. "Renegotiating acquired rights in the oil and gas industries: Industry and political cycles meet the rule of law." Journal of World Energy Law and Business 1 (1): 55-97. 